\title{
IMPROVING PHYSICIAN ENGAGEMENT IN \\ INTERPROFESSIONAL COLLABORATIVE PRACTICE \\ IN RURAL EMERGENCY DEPARTMENTS
}

Kelly Might Wilson

\begin{abstract}
A DNP project submitted to the faculty at the University of North Carolina at Chapel Hill in partial fulfillment of the requirements for the degree of Doctor of Nursing Practice in Healthcare Systems - Outcomes Management in the School of Nursing.
\end{abstract}

Chapel Hill

2017

Approved by:

Jennifer Leeman

Donna Havens

Barry Saunders 
(C) 2017

Kelly Might Wilson

ALL RIGHTS RESERVED 


\begin{abstract}
Kelly Might Wilson: Improving Physician Engagement in Interprofessional Collaborative Practice in Rural Emergency Departments

(Under the direction of Jennifer Leeman)
\end{abstract}

Introduction. Poor communication and collaboration among healthcare professionals negatively impact patient outcomes and quality of care. Due to the nature of the care environment, emergency departments (EDs) are particularly susceptible to this problem. Developing interprofessional collaborative practice (IPCP) is critical to managing complexity and improving ED care. One factor limiting efforts to create IPCP is the low level of physician engagement. The purpose of this project was to explore strategies implemented by rural EDs to promote physician participation in IPCP.

Methods. The setting for this work was a HRSA-sponsored project, Shaping Systems, which involved a quality improvement collaborative designed to promote nursing's capacity to develop IPCP in the ED setting of four rural North Carolina hospitals. Qualitative data were collected through participation in collaborative meetings, site visits, and structured interviews with participating site team members. Data were reviewed using content analysis, coded, and analyzed across cases to identify a list of strategies and report contextual factors related to improving physician engagement.

Results. Strategies for engaging physicians were found at all participating sites with similar strategies noted at multiple sites. Successful approaches were grouped into structural, logistical, interpersonal, and quality improvement related strategies. Contextual factors that 
served as barriers or facilitators of these strategies were also reported. The result is a toolkit that includes strategies as well as the contextual factors that influence their applicability and success.

Discussion/Conclusion. The results of this project were shared with participating EDs both to confirm their validity and so they could learn from each other to improve physician engagement in their efforts to create IPCP and improve the quality of care in their settings. Nursing leaders and others can use this toolkit as needed to address similar issues with physician engagement in their EDs. 


\section{ACKNOWLEDGEMENTS}

I would like to thank my family for all the support they have given me throughout this project. My husband and children have provided me with both time and encouragement to work on this project. My parents have also given me the support I needed to successfully accomplish my goals. I am grateful to Dr. Jennifer Leeman, the chair of my DNP project committee, for her valuable guidance throughout this project. She made both herself and her expertise available to me whenever needed. I appreciate the time and support from her and my committee members, Dr. Donna Havens and Dr. Barry Saunders. I also want to thank Dr. Havens for allowing me to work with her project as well as all the members of the Shaping Systems team from UNC. And finally, I am grateful to the Shaping Systems site team members for welcoming me into their quality improvement collaborative and their hospitals. I appreciate their willingness to share all the tremendous work they are doing to improve the care they provide in their EDs.

\section{Source of funding}

This project was supported by funds from the Division of Nursing (DN), Bureau of Health Professions (BHPr), Health Resources and Services Administration (HRSA), and the Department of Health and Human Services (DHHS) under D11 HP09752 "Spiraling Upward for Nurse Retention and Quality Care" (DS Havens, Principal Investigator). The information and content or conclusions presented in this project are those of the author and should not be construed as the official position or policy, neither should any official endorsement be inferred by the DN, BHPr, HRSA, DHHS or the US government. 


\section{TABLE OF CONTENTS}

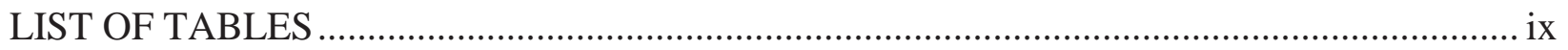

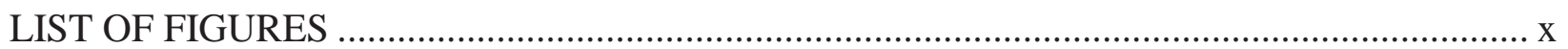

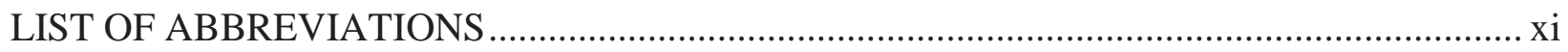

CHAPTER 1: BACKGROUND AND SIGNIFICANCE............................................................. 1

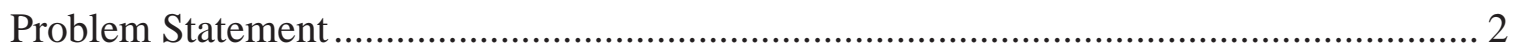

Physicians do not engage in efforts to improve communication and collaboration in EDs ..................................................................................... 2

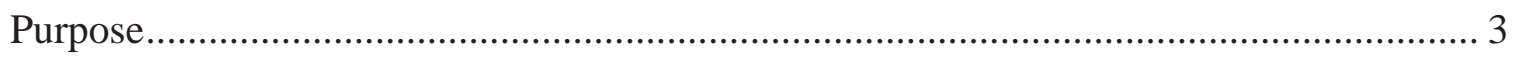

Practice Question ..................................................................................................... 3

CHAPTER 2: REVIEW OF THE LITERATURE ………………........................................... 4

Communication and Collaboration in EDs ..................................................................... 4

Consequences of Poor Communication and Collaboration ........................................... 4

Impact of the ED Environment.............................................................................. 6

Additional Barriers to Interprofessional Communication and

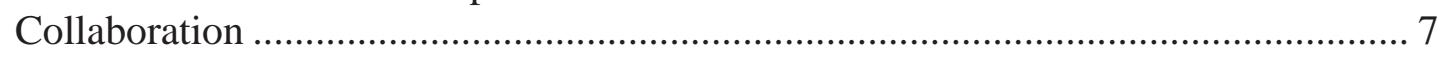

Interprofessional Collaborative Practice (IPCP)………….............................................. 8

Who Should Be Involved................................................................................. 9

Physician Engagement ............................................................................................... 10

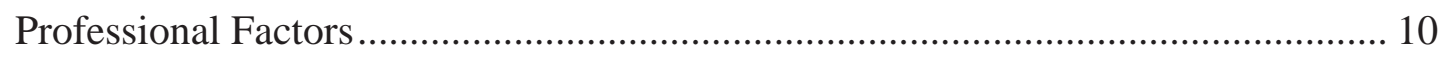

Additional Factors ……………………........................................................... 12

Strategies for Improving Physician Engagement ........................................................ 13

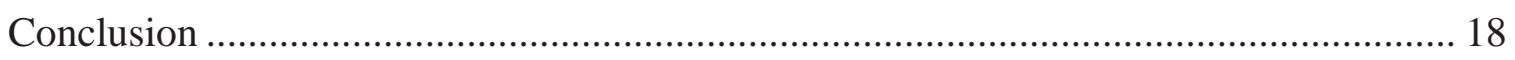


CHAPTER 3: CONCEPTUAL AND THEORETICAL FRAMEWORK.................................. 19

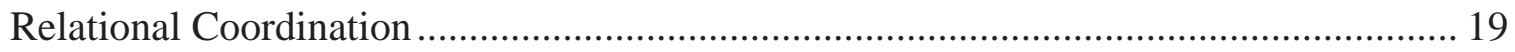

Relational Coordination as a Mutually Reinforcing Cycle of Relationships and Communication ........................................................................... 21

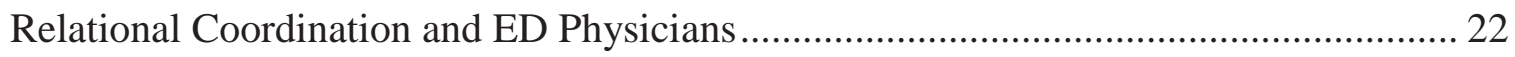

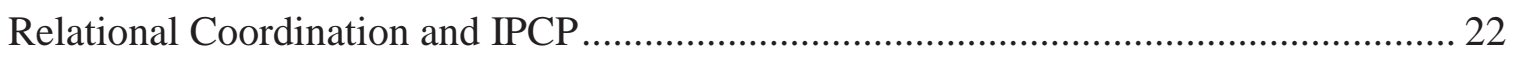

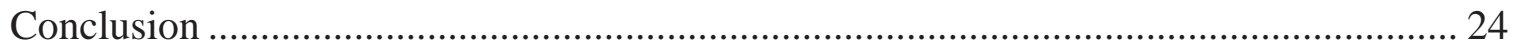

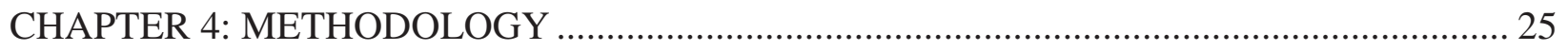

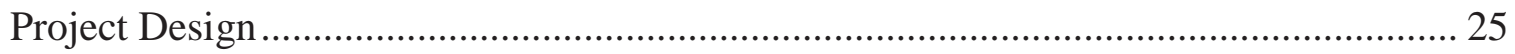

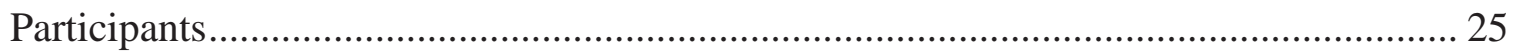

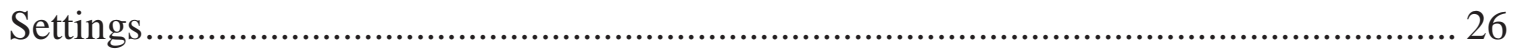

Shaping Systems Grant Project .................................................................. 26

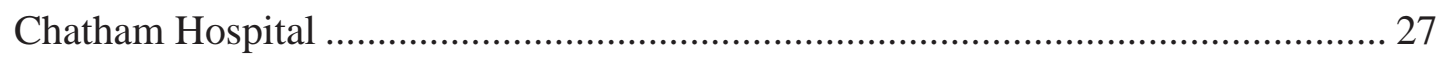

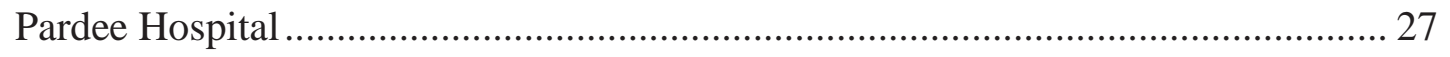

Southeastern Medical Center ............................................................................. 27

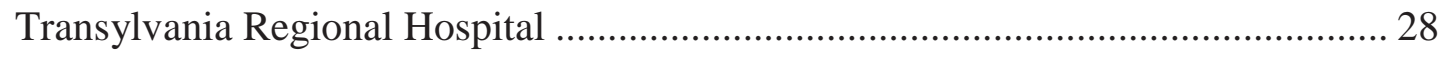

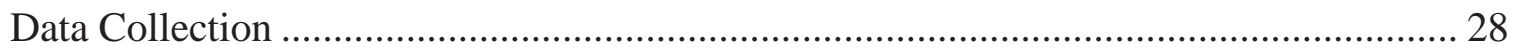

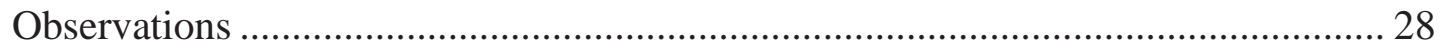

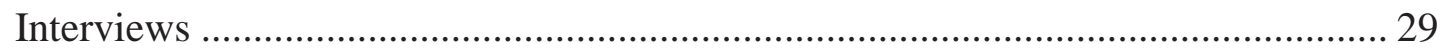

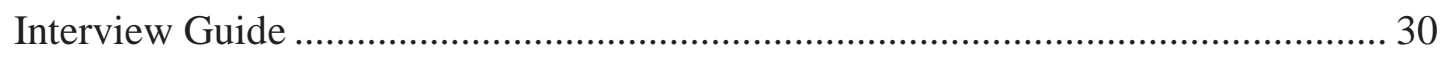

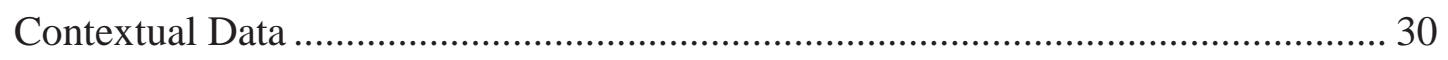

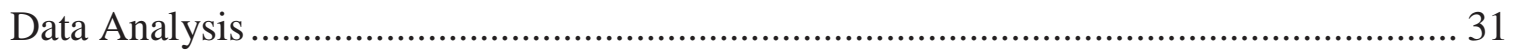

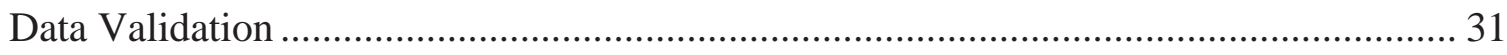

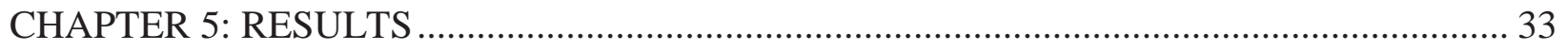

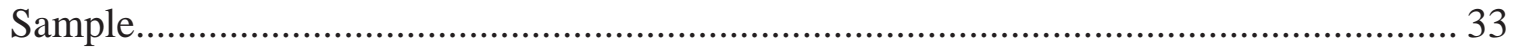


Findings on Physician Engagement in Interprofessional Collaborative

Practice Activities ...................................................................................................... 38

Contextual Factors Influencing Physician Engagement .............................................. 38

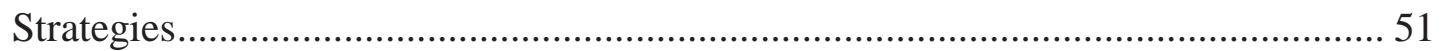

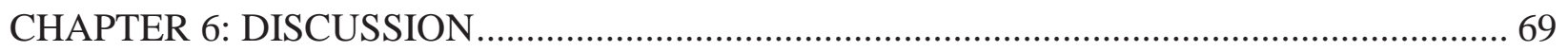

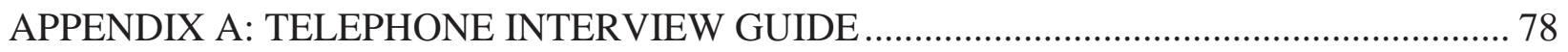

APPENDIX B: TOOLKIT FOR IMPROVING PHYSICIAN ENGAGMENT............................. 80

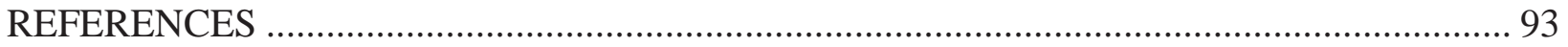




\section{LIST OF TABLES}

Table 1. Participants in the 5 learning collaboratives associated with this project...................... 34

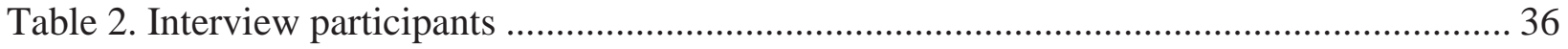

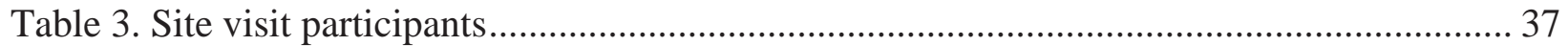

Table 4. Projects, initiatives, collaboratives, issues discussed by ED site teams ...................... 43

Table 5. Examples of meetings used to engage physicians in IPCP..................................... 52

Table 6. Summary of successful strategies for managing and communicating

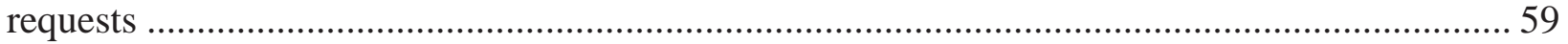

Table 7. Approaches to building personal relationships with physicians ................................ 61

Table 8. Examples of other professionals used to improve physician engagement..................... 64 


\section{LIST OF FIGURES}

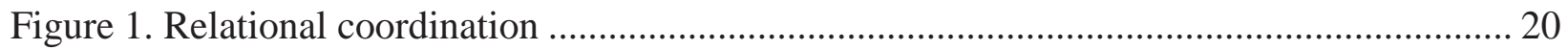

Figure 2. Relational coordination model ............................................................................ 21

Figure 3. Framework for improving physician engagement in IPCP ................................... 70 


\section{LIST OF ABBREVIATIONS}

$\begin{array}{ll}\text { APP } & \text { Advanced Practice Provider } \\ \text { CDC } & \text { Centers for Disease Control } \\ \text { DNP } & \text { Doctor of Nursing Practice } \\ \text { ED } & \text { Emergency Department } \\ \text { EHR } & \text { Electronic Health Record } \\ \text { FMEA } & \text { Failure Mode and Effects Analysis } \\ \text { HRSA } & \text { Health Resources and Services Administration } \\ \text { IOM } & \text { Institute of Medicine } \\ \text { IPCP } & \text { Interprofessional Collaborative Practice } \\ \text { RC } & \text { Relational Coordination } \\ \text { SBAR } & \text { Situation-Background-Assessment-Recommendation } \\ \text { TeamSTEPPS } & \text { Team Strategies and Tools to Enhance Performance and Patient Safety } \\ \text { UNC } & \text { University of North Carolina at Chapel Hill } \\ \text { WHO } & \text { World Health Organization }\end{array}$




\section{CHAPTER 1: BACKGROUND AND SIGNIFICANCE}

Emergency departments (EDs) in the United States treat an increasing number of patients each year (Weiss, Wier, Stocks, \& Blanchard, 2014). In 2013, 130.4 million patient visits were made to Emergency Departments (Centers for Disease Control and Prevention [CDC], 2017). ED patients are older, sicker, and require more complex care than in the past (Greenwald et al., 2015; Herring et al., 2013; Pines, Mullens, Cooper, Feng, Roth, 2012), which contributes to problems with the quality and efficiency of ED care (Institute of Medicine [IOM], 2007; Mullins \& Pines, 2014). In their 2007 document, Hospital-Based Emergency Care: At the Breaking Point, the Institute of Medicine acknowledged the challenges faced by EDs as well as the need for improved communication and collaboration among healthcare professionals in these settings.

Poor communication and collaboration among healthcare professionals negatively impact patient outcomes and quality of care in EDs (Bagnasco et al., 2013; Flowerdew, Brown, Vincent, \& Woloshynowych, 2012; Hitchcock, Gillespie, Crilly, \& Chaboyer, 2014; Kilner \& Sheppard, 2010; Redfern, Brown, \& Vincent, 2009). The often chaotic, busy, and stressful environment of the ED exacerbates communication and collaboration problems (Eisenberg et al., 2005;

Flowerdew, Brown, Russ, Vincent, \& Woloshynowych, 2012; Varjoshani, Hosseini, Khankeh, \& Ahmadi, 2014). Overcrowding, long wait times, large numbers of patients leaving without being seen, and clinical errors have all been linked to ineffective or insufficient interdisciplinary communication and collaboration in EDs (IOM, 2007). Issues such as ED overcrowding lead to decreased quality of hospital care (Mullins \& Pines, 2014). 
Improving interprofessional communication and collaboration is critical to managing complexity and improving care in the ED (IOM, 2007). To address this problem, the Heath Resources and Services Administration (HRSA) is funding projects to improve interprofessional practice, including Dr. Donna Haven's “Shaping Systems to Promote Desired Outcomes: Interprofessional Collaborative Practice in Rural North Carolina EDs (Shaping Systems), " an initiative involving four North Carolina EDs. Interprofessional collaborative practice (IPCP) occurs when members of multiple healthcare professions work together with patients, families, and communities to provide high quality patient care (World Health Organization [WHO], 2010). Collaborative practice can impact the "triple aim" outcomes that include improved patient experience, improved population health, and reduced health care costs (Berwick, Nolan, \& Whittington, 2008; Lutfiyya, Brandt, Delaney, Pechacek, \& Cerra 2015).

\section{Problem Statement}

\section{Physicians do not engage in efforts to improve communication and collaboration in EDs}

Despite evidence that suggests IPCP positively affects patient outcomes as well as recommendations that EDs adopt IPCP, many healthcare organizations find it difficult to create IPCP environments in their settings (IOM, 2007; Lutfiyya et al., 2015; Zwarenstein, Goldman, \& Reeves, 2009). One factor limiting current efforts to improve communication and collaboration is the low level of physician engagement (Barker, Bosco, \& Oandasan, 2005; PoochikianSarkissian et al., 2008). The rural EDs participating in the HRSA-funded Shaping Systems project have experienced difficulty getting physicians to participate in project activities or local efforts to improve IPCP in their EDs.

Physician commitment is necessary for change to a sustainable collaborative culture (Cote, Lauzon, \& Kyk-Strickland, 2008). The challenge now is to identify evidence-based strategies that organizations can use to overcome this obstacle (Tremblay et al., 2010). 
Exploring strategies to engage physicians in IPCP is part of the larger need for research on how IPCP is successfully implemented, including context-specific experiences (Lutfiyya et al., 2015). Understanding and addressing the barriers to physician engagement in interprofessional practice will also benefit healthcare organizations as they develop and implement solutions to improve patient care in their EDs.

\section{Purpose}

The purpose of this DNP project was to explore strategies that rural EDs implemented to promote physician participation in IPCP. The project built on Dr. Donna Haven's Shaping Systems project in four rural EDs in North Carolina. Her project involved bringing these organizations together in a learning collaborative to work with and learn from each other while developing IPCP in their settings. Participants from all sites taking part in this quality improvement collaborative reported difficulty getting physicians to participate in collaborative activities or to engage in local efforts to improve IPCP in their EDs. This DNP project documented and synthesized challenges, needed resources, and successful strategies used to improve physician participation in local activities that promote IPCP in each of the four sites that participated in the quality improvement collaborative. This information was shared among the sites so that successful strategies could be replicated. The final product is a toolkit that can be used to foster physician engagement in IPCP in these EDs and other rural EDs.

\section{Practice Question}

What are barriers, facilitators, needed resources, and successful strategies to improve physician engagement in interprofessional collaborative practice in four rural EDs participating in a project designed to build IPCP capacity? 


\section{CHAPTER 2: REVIEW OF THE LITERATURE Communication and Collaboration in EDs}

The need to improve communication and collaboration among healthcare professionals in the ED has been identified by the Institute of Medicine (2007) as well as other researchers and ED staff members (Cameron et al., 2010; Creswick, Westbrook, \& Braithwaite, 2009; Parsons, Cornett, \& Burns, 2005). Effective interprofessional communication is necessary for successful collaboration and/or teamwork and both play an important role in patient care in the ED (Hitchcock et al., 2014; Kilner \& Sheppard, 2010; Patterson et al., 2013; Redfern et al., 2009). Researchers have linked failures in communication and/or collaboration to quality and safety issues in this setting (Bagnasco et al., 2013; Cosby et al., 2008; Flowerdew, Brown, Vincent, et al., 2012). Examination of how interprofessional communication and collaboration occurs in EDs illustrates how the ED environment contributes to this problem and reveals additional barriers to making improvements (Eisenberg et al., 2005; Fairbanks, Bisantz, \& Sunm, 2007; Flowerdew, Brown, Russ, et al., 2012; Hitchcock et al., 2014; Kilner \& Sheppard, 2010; Redfern et al., 2009).

\section{Consequences of Poor Communication and Collaboration}

There are several negative consequences of poor interprofessional communication and collaboration in ED settings, including clinical errors and unfavorable patient and/or staff outcomes (Bagnasco et al., 2013; Flowerdew, Brown, Vincent, et al., 2012). Clinical errors and adverse events in the ED have been linked to communication and teamwork failures (Cosby et al., 2008; Flowerdew, Brown, Vincent, et al., 2012; Fordyce et al., 2003; Juarez et al., 2009; 
Redfern et al., 2008; Risser et al., 1999). Cosby et al. (2008) identified teamwork as a contributing factor to $61 \%$ of patient care errors while Fordyce et al. (2003) categorized $19 \%$ of errors as related to communication or team functioning. In a retrospective study, Risser et al. (1999) found that teamwork failure contributed to clinical error in at least $20 \%$ of cases reviewed. A national study of the characteristics of voluntarily reported ED medication errors found that poor communication was one of the leading causes of medication errors in this setting (Pham et al., 2011).

High-risk industries, such as aviation and nuclear energy, have recognized the importance of communication and collaboration in safety. Methods used in healthcare to identify communication and teamwork safety failures, such as the Failure Mode and Effects Analysis (FMEA), were developed in the aviation industry. A failure mode is a way in which something might fail, potentially causing an error (Redfern et al., 2009). Bagnasco et al. (2013) found that the most dangerous failure modes were related to communication among health professionals, and Redfern et al. (2009) reported that each communication step and event in the ED patient pathway had at least one failure mode. Healthcare organizations have begun to adopt models developed by these industries for improving communication and teamwork, such as SituationBackground-Assessment-Recommendations (a standardized communication tool) and TeamSTEPPS (a team training model) (Bagnasco et al., 2013; Redfern et al., 2009; Risser et al., 1999).

Ineffective communication and collaboration also contribute to inefficiencies that may result in delays, long wait times, and overcrowding for patients in EDs (Eisenberg, Baglia, \& Pynes, 2006; Hitchcock et al., 2014; Redfern et al., 2009). These consequences may result in larger numbers of patients leaving without being seen. Overcrowding may be both a 
consequence of and contributor to communication and collaboration problems in the ED (Eisenberg et al., 2005). Although these problems may not cause direct errors in patient care, they can negatively impact patient safety and satisfaction. More research that specifically investigates the impact of poor communication and collaboration on these ED outcomes is needed (Kilner \& Sheppard, 2010; Patterson et al., 2013). Promoting teamwork and communication in the ED may also positively affect staff satisfaction (Kilner \& Sheppard, 2010). According to Parsons et al. (2005), interprofessional communication and relationships are described by staff as necessary for a healthy ED workplace. Additional benefits would include reductions in staff burnout and turnover. Pruitt and Liebelt (2010) note that effective teamwork would not only impact safety, it would also lead to more effective and efficient patient care. Improvements in interprofessional communication and collaboration would impact a number of problems that EDs are currently experiencing.

\section{Impact of the ED Environment}

The unique ED environment contributes to suboptimal communication and collaboration among healthcare providers in this setting. The conditions under which healthcare professionals work in the ED make it a high-risk setting (Redfern et al., 2009). Barriers to better communication in the ED include unpredictable and often high work and communication loads (Cameron et al., 2010). Communication and collaboration are negatively affected by the physical environment in this setting, including lack of dedicated space for private communication (Eisenberg et al., 2005; Reddy \& Spence, 2006) and high noise levels (Slade, 2015). Multitasking and interruptions have been found to contribute to the high-risk nature of the ED environment (Kilner \& Sheppard, 2010; Spencer, Coiera, \& Logan, 2004; Woloshynowych, Davis, Brown, \& Vincent, 2007). Chisholm, Collison, Nelson, and Cordell (2000) found that ED physicians were interrupted 10.3 times per hour, while Coiera, Jayasuriya, Hardy, Bannan, and 
Thorpe (2002) classified one-third of communication events in an ED as interruptions. Pressure and stress that result from time constraints, overcrowding and need for accuracy have also been found to inhibit effective communication and collaboration in the ED (Rasmussen et al., 2014; Varjoshani et al., 2014). This high-risk ED environment lends itself to using aviation safety research on communication and teamwork, including Crew Resource Management (Molesworth \& Estivalb, 2015; Murphy, 2001; Pruitt \& Liebelt, 2010). Many factors specific to the ED environment make successful interprofessional communication and collaboration difficult to achieve.

\section{Additional Barriers to Interprofessional Communication and Collaboration}

Several barriers to interprofessional communication and collaboration observed in other healthcare settings are also present in the ED. Dynamics related to leadership, personal relationships, and professional roles impede good communication and collaboration between healthcare professionals. Flowerdew, Brown, Russ, et al. (2012) found that leadership is a key component of team function. Unlike other disciplines, such as the military, from which healthcare has adopted several teamwork strategies, a more flexible communication structure is sometimes needed (Jacobsson, Hargestam, Hultin, \& Brulin, 2012; Klein, Ziegert, Knight, \& Xiao, 2006). Personal relationships can also play a role in how professionals work together in an ED setting. Differences in the temperaments of nurses that work in EDs versus other units, misunderstandings and feelings of mistrust, and lack of respect have been cited as factors affecting communication and collaboration among professionals within and between the ED and other hospital departments (Cameron et al., 2010; Eisenberg et al., 2006; Flowerdew, Brown, Russ, et al., 2012).

The traditional roles and separate education of healthcare professionals leads to independent, functional work structures (often referred to as 'silos') (Eisenberg et al., 2005; 
Kilner \& Sheppard, 2010). The result of these 'silos' is often a lack of collaboration with other healthcare team members. Individuals are more closely connected to and communicate most with colleagues in their own professional groups (Creswick et al., 2009; Patterson et al., 2013). Research in business and organizational management has resulted in similar findings in these disciplines and has guided the study of communication relationships in ED settings using social network analysis (Cross \& Sproull, 2004; Manev \& Stevenson, 2003). Creswick et al. (2009) reported that ED staff believed that improved communication across professional groups would result in fewer medication errors. Hierarchical aspects of functional roles can also lead to intimidation that results in lack of communication (Eisenberg et al., 2005; Flowerdew, Brown, Vincent, et al., 2012; Juarez et al., 2009). According to Risser et al. (1999), lack of crossmonitoring among professionals was a factor in many malpractice incidents in the ED. Interpersonal relationships and professional roles can be barriers to improving communication and collaboration in the ED.

\section{Interprofessional Collaborative Practice (IPCP)}

To improve communication and collaboration among healthcare professionals in the ED setting, all involved disciplines need to change the way they practice (Bagnasco et al., 2013; Redfern et al., 2009). Observation in five different EDs found disciplines working side by side but little evidence of interdisciplinary collaboration (Slade, 2015). According to Pruitt and Liebelt (2010), professionals in the ED work alongside one another in a group when they should be working with each other as a team with common goals, complementary skills, and shared accountability. By definition, IPCP calls for healthcare professionals to work with each other in this way (WHO, 2010). Therefore, improvements in the IPCP environment can positively impact this problem. 


\section{Who Should Be Involved}

Healthcare team structures can differ and include a variety of professions or disciplines (Jacobsson et al., 2012). Organizational factors are often important in coordinating clinical care in the ED and may even require communication and coordination with non-clinical professionals, such as unit secretaries and security personnel (Reddy \& Spence, 2006). Contextual features of hospitals, such as academic vs. community or rural vs. urban, will affect the composition of an interprofessional healthcare team as well as their needs regarding efforts to improve communication and collaboration (Fairbanks et al., 2007; Reddy \& Spence, 2006). Lack of cross-functional communication and collaboration within the hospital system (e.g. between ED and lab department) has also been reported (Cosby, 2003; Flowerdew, Brown, Russ, et al., 2012). Eisenberg et al. (2006) found a lack of "system consciousness" regarding emergency care. The need for more research related to the understanding of ED staff needs by other hospital units or departments has been noted (Varjoshani et al., 2014). Further research on the importance of using a team or contextual approach when evaluating communication practices in the ED setting is needed (Cameron et al., 2010; Eisenberg et al., 2006; Kilner \& Sheppard, 2010).

Improving the IPCP environment in EDs will address many of the barriers to effective communication and collaboration in these settings. Using a contextual approach to implementing strategies to improve IPCP will be important. Failures in communication and collaboration involve a variety of professionals, disciplines, and systems; therefore, many different types of staff members should be involved in solutions to these problems (Cameron et al., 2010; Eisenberg et al., 2006; Redfern et al., 2009). Physicians are key members of patient care teams in EDs and their involvement in efforts to improve IPCP is necessary (Lindgren, Baathe, \& Dellve, 2012). However, physician engagement in collaborative practice is 
challenging for organizations (Barker et al., 2005; Kreindler, Dowd, Dana Star, \& Gottschalk, 2012; Poochikian-Sarkissian et al., 2008).

\section{Physician Engagement}

Differences in professional roles have been identified as barriers to effective communication and collaboration in the ED setting (Eisenberg et al., 2005; Kilner \& Sheppard, 2010; Slade, 2015). The traditional role of physicians as autonomous professionals contributes to their lack of participation in collaborative practice as well as organizational initiatives aimed at improving the delivery of healthcare services (Baathe \& Norback, 2013; Lindgren et al., 2012; Poochikian-Sarkissian et al., 2008). Human behavior is influenced by a person's level of association or affiliation with their profession and the organization in which they work (Fitzgerald \& Teal, 2004). Strategies for engaging physicians in organizational improvement need to address physicians not only as individuals, but also as members of a profession with given attributes (Kreindler et al., 2014; Stevens, 2013). The literature has identified issues related to physicians' professional identity as well as other obstacles to successful physician engagement (Kaissi, 2014). Barriers to physician engagement in hospital or organizational activities, such as quality or process improvement initiatives, are similar to challenges related to physician participation in collaborative practice and teamwork. Strategies for improving physician engagement in organizational initiatives can inform potential approaches to increase physician participation in collaborative practice.

\section{Professional Factors}

Factors associated with the medical profession, such as autonomy, power, and status, have been associated with a lack of physician participation in collaboration, communication, and efforts to improve healthcare delivery. Physicians understand their profession to be one in which decisions are made autonomously, using their own judgement (Baathe \& Norback, 2013; 
Whitehead, 2007). The result has been a tradition of physician-centered practice that values independence over teamwork (Taitz, Lee, \& Sequist, 2012). Characteristics of physician culture include independence and autonomy as well as their perception that they are "captains of the ship" and "teamwork is fine, but they are in charge" (Byrnes, 2015, p. 40). Unequal power and status among different healthcare professions have traditionally led to a strict hierarchical structure with physicians at the top (Poochikian-Sarkissian et al., 2008; Whitehead, 2007). Issues related to these power differences, such as conflict and intimidation, negatively impact interprofessional collaboration and communication (Herbert et al., 2007; Manojlovich, 2010; Tang, Chan, Zhou, \& Liaw, 2013). Power and status differences must be recognized to improve physician engagement in collaborative practice or teamwork (Herbert et al., 2007; Kreindler et al., 2012; Meleis, 2016; Whitehead, 2007).

The autonomous physician role has been associated with less engagement in healthcare development (Lindgren et al., 2013). Healthcare professionals have expressed a preference for a less rigid hierarchy (Herbert et al., 2007); however, efforts to restructure healthcare systems are often seen as a threat to physicians that reduces power and autonomy over their clinical practice (Kaissi, 2014). Changes in the healthcare system that call for more focus on effectiveness and efficiency in patient care management and reimbursement tied to value have contributed to physicians' feelings of loss of control and autonomy (Rosenstein, 2015). Many physicians "just want to practice medicine" and are less interested in changes that lead to more integrated roles and responsibilities as well as frustrated by accountability for new performance and care measures (Rosenstein, 2015, p.113). Changes occurring in the healthcare delivery and payment systems have contributed to increased levels of stress and burnout in physicians (Rosenstein, 2015; Shanafelt \& Noseworthy, 2017). Physician burnout has been noted to result in loss of 
engagement and commitment to organizational efforts to improve collaborative care (Rosenstein, 2015; Shanafelt \& Noseworthy, 2017; Swenson, Kabcenell, \& Shanafelt, 2016). However, physicians are essential members of the care team needed to improve healthcare patient service delivery and improved quality of care (Skillman et al., 2017).

\section{Additional Factors}

Challenges related to engaging physicians in interprofessional collaborative practice include employment status, training, time, and attitudes toward collaboration. Physicians who are employed by a hospital feel more connected to the organization and collaborate more with other hospital staff as compared to those who are not (Fitzgerald \& Teal, 2003). Hospitalphysician alignment structures often do not promote physician engagement in quality improvement initiatives and many physicians do not recognize their responsibility for hospital quality (Suelflow, 2016). Physician participation in improvement processes has been linked to workplace continuity (Lindgren et al., 2013). Physician relationships to the organization were found to affect physician engagement in new health care innovation models (Skillman et al., 2017). Physicians that were considered "internal" to the organization were more likely to engage in and play a role in policies and practices aimed at physician engagement than "external" physicians (Skillman et al., 2017).

Lack of engagement in collaboration and organizational improvement are also influenced by training and education. Most physicians were educated with a focus on technical training and medical competence development rather than skills related to group dynamics or collaboration (Baathe \& Norback, 2013; Manojlovich, 2010; Tang et al., 2013). Younger physicians collaborated more often because they trained alongside other professionals and valued their input (Fitzgerald \& Teal, 2003). Rosenstein (2015) suggests that due to the increasing importance of team collaboration, the process of physician engagement should begin in medical school with 
instruction in business and personal development skills. Similarly, physicians' prior experiences have been found to affect their engagement in new health care innovation programs (Skillman et al., 2017).

Time has been identified as a challenge to working in a collaborative model (Herbert et al., 2007; Poochikian-Sarkissian et al., 2008) as well as to physician engagement in organizational quality and safety efforts (Taitz et al., 2012). Lack of time has been linked to work environment and competing patient care responsibilities (Lindgren et al., 2013). Physician concerns over time and effort required were also noted as barriers to physician engagement in new health care delivery innovation programs (Skillman et al., 2017).

Physicians' perceptions of communication and collaborative work differ from those of nurses (Manojlovich, 2010). Nurses express more of a desire to work collaboratively than physicians (Tang et al., 2013). Collaborative relationships are also a more important component of job satisfaction for nurses than for physicians (Hojat et al., 2001). When asked what is important to feeling engaged, physicians ranked good relationships with other physicians higher than good relationships with non-physician clinical staff (Whitlock \& Stark, 2014).

\section{Strategies for Improving Physician Engagement}

The development of organizational structures and work practices can be successful in supporting more positive group relations, interprofessional collaboration, and physician engagement (Kaissi, 2014; Kreindler et al., 2012; Lindgren et al., 2013; Pilon et al., 2015). The need for formal policies or procedures to implement interprofessional practice was identified by one interprofessional practice team (Poochikian-Sarkissian et al., 2008). Examples of structural solutions for improving physician participation in collaborative practice include organizational strategies such as interprofessional education programs, interprofessional ward rounds (Tang et al., 2013), communication protocols, joint workspaces (Manojlovich, 2010; Morgan, Pullon, \& 
McKinlay, 2015), interprofessional meetings (Minamizono et al., 2013), and scheduling that supports workgroup continuity (Kaissi, 2014; Lindgren et al., 2013). Improving collaboration can involve shared structures while still maintaining separate, distinct professional groups (Kreindler et al., 2012). Similarly, successful strategies identified for removing barriers to physician engagement in hospital quality initiatives or new health care delivery programs include training, outreach, tailored education and regular meetings (Suelflow, 2016). Training specifically for improving communication and team collaboration skills has also been suggested to help physicians become more engaged in their organizations (Rosenstein, 2015).

Leadership and management play an important role in promoting physician engagement. Support by managers can foster understanding and interdependence between professional identities (Fitzgerald \& Teal, 2003). Leadership support sets the tone, creates a culture of collaboration, and establishes appropriate infrastructure (Liebhaber, Draper, \& Cohen, 2009). Management structures should also be collaborative, support team development, and provide feedback (Morgan et al., 2015). A positive physician-organization relationship is important for reducing physician burnout which leads to a loss in engagement (Swenson et al., 2016). This relationship can be developed by using participative management and developing physician leaders (Swenson et al., 2016). Collaborative management requires identifying and developing physician leaders trained to work collaboratively (Duberman, Hemker, \& Soloman, 2015; Kaissi, 2014). Allowing physician leaders to lead quality initiatives was identified as a successful strategy for engaging physicians (Suelflow, 2016).

The identification of physician champions is another strategy for increasing physician engagement in organizational initiatives (Kaissi, 2014; Kreindler et al., 2014; Suelflow, 2016). Use of a physician champion involves recruiting a physician to serve as "a thought leader who 
can guide the process and encourage participation (Whitlock \& Stark, 2014, p.11). Physician champions should be informed of the project first and consulted about who and how to engage other physicians (Sears, 2011). Physician champions should be highly respected in their area and possess personality traits such as a willingness to change practices as well as the ability to influence others (Liebhaber et al., 2009). For implementing new health care delivery innovations, these physicians are used to educate and encourage buy-in from other physicians (Skillman et al., 2017). Organizations should work informally with and through these individuals to encourage physician engagement (Guthrie, 2005).

Clear and efficient communication with physicians has been suggested as a strategy for enhancing physician engagement in organizational activities (Kaissi, 2014; Suelflow, 2016). Communication can be used to ensure physicians can find opportunities to engage (Kaissi, 2014) and reduce the effort needed to participate (Suelflow, 2016). Clear communication and effective messaging can also convey the importance of improvement projects as well as their potential impact on issues such as patient safety and improved efficiency. One-on-one meetings, emails, newsletters, and posters can be used for communication. (Liebhaber et al., 2009). Open communication and allowing physicians to voice their thoughts and concerns are also critical factors for successful physician engagement (Rosenstein, 2015; Spaulding, Gamm, \& Menser, 2014). Regular communication between physicians and other care team members can be used to adjust to physicians' needs and preferences (Skillman et al., 2017).

The ability to find and communicate a common purpose and shared goals is useful for encouraging physician engagement (Kaissi, 2014; Kreindler et al., 2012). One way to use shared goals to improve physician engagement involves formal alignment strategies (Suelflow, 2016). These strategies include formal contracts, mutual accountability for outcomes, standardized 
documentation and communication, or hospital employment (Suelflow, 2016). Employing physicians has been associated with improved physician engagement in organizational improvement (Fitzgerald \& Teal, 2003; Stevens, 2013). Physician employment by an organization helps with incentives and achieves an alignment in the quality agenda between physicians and hospitals (Liebhaber et al., 2009).

Finding a common purpose can also be done less formally. The desire to improve patient satisfaction and outcomes is an example of a goal that would be mutually rewarding (Guthrie, 2005). Leaders at two different major health systems reported success in engaging physicians in their organizational initiatives by connecting projects to patient related problems (Duberman et al., 2015; Spaulding et al., 2014). One of the key characteristics of physician culture is that patient welfare is their top concern (Byrnes, 2015). This characteristic can be leveraged to encourage physician engagement in efforts geared at improving the quality of patient care (Byrnes, 2015). Physicians will also be more motivated to engage in efforts if they are told how these efforts will reduce their own frustrations, make their lives easier, or improve efficiencies that will allow them to spend more time providing patient care (Rosenstein, 2015). Successful physician engagement was also found to occur when programs explained the benefits of new health delivery innovations, such as workflow efficiencies (Skillman et al., 2017).

Making these connections may require the use of meaningful and reliable data related to patient and staff processes and outcomes. Using data is another strategy for encouraging physician participation in organizational initiatives (Kaissi, 2014; Liebhaber et al., 2009; Sears, 2011). As trained scientists, being data-driven is a characteristic of physician culture (Byrnes, 2015). Meaningful data can be used to make physicians aware of problems (Kaissi, 2014). Reporting is one strategy for giving feedback on quality metrics (Suelflow, 2016). This strategy 
can involve public or private reporting and may include comparative data in the form of a scorecard (Suelflow, 2016). Sharing data was noted to be important to physicians participating in new healthcare delivery innovation programs; however, the inability to provide timely results was a challenge to building relationships with some physicians (Skillman et al., 2017).

Intrinsic and extrinsic incentives have been suggested as potential motivations for physicians to engage in organizational improvement processes, including interprofessional collaborative practice. Physicians will often choose to participate in activities that serve as sources of professional fulfillment and learning (Lindgren et al., 2013). According to physicians surveyed about engagement, feeling engaged is very important to their job satisfaction (Stark, 2014). Recognition for their participation in organizational activities can also encourage physician engagement (Baathe \& Norback, 2013; Kaissi, 2014). Using public reporting to share only commendable data through public awards or recognition events has been used as a strategy to improve physician engagement (Suelflow, 2016).

Compensation and/or dedicated time for participating in organizational initiatives have also been identified in the literature as helpful for improving engagement (Kaissi, 2014; Liebhaber et al., 2009; Poochikian-Sarkissian et al., 2008). Lack of payment for work performed was identified as a barrier that could be addressed by providing stipends, salary, or grants (Suelflow, 2016). Financial incentives were found to result in the improved engagement of resident and fellow physicians in quality initiatives; however, it was noted that without systems support, these incentives were not as successful (Suelflow, 2016). Systems support included dedicated time, altering work schedules, and administrative support from quality experts (Suelflow, 2016). Using financial incentives to promote physician engagement in healthcare innovation programs was reported to have mixed results (Skillman et al., 2017). 
Strategies that involved climate or culture around physician engagement have been reported (Rosenstein, 2015; Skillman et al., 2017; Suelflow, 2016). Efforts to promote attitudinal and cultural changes toward quality initiatives among physicians and hospitals have been suggested (Suelflow, 2016). Given the increased need for team collaboration in healthcare settings, the idea that organizations should address the "cultural fit" of physicians with regard to goals and expectations has been recommended (Rosenstein, 2015).

Taking physicians' needs and concerns into account has been raised as an important component to engaging physicians (Skillman et al., 2017; Swenson et al., 2016; Stark, 2014). Listening to physician concerns and balancing their need for some control over their work are recommended for promoting physician engagement (Swenson et al., 2016). Effective strategies for engaging physicians in healthcare innovation programs included addressing physicians' concerns and adapting to their needs and preferences (Skillman et al., 2017). Hospitals should make efforts to engage in the physician's agenda (Suelflow, 2016). Stark (2014) suggests that increasing physician engagement should begin by discovering what is important to them.

\section{Conclusion}

Failures in communication and collaboration involve a variety of professionals, disciplines, and systems; therefore, different types of staff members should be involved in solutions to these problems (Cameron et al., 2010; Eisenberg et al., 2006; Redfern et al., 2009). Improving IPCP in EDs can address many of the barriers to effective communication and collaboration. Physician participation is needed to create a successful IPCP environment (Cote et al., 2008). Using a contextual approach to understand physician engagement and implement strategies is important for successfully improving IPCP in these settings (Kaissi, 2014; Ward, Baloh, Zhu, \& Stewart, 2016). 


\section{CHAPTER 3: CONCEPTUAL AND THEORETICAL FRAMEWORK Relational Coordination}

High quality communication and collaboration among healthcare professionals can be understood using the theory of relational coordination. This theory explains the relational dynamics that are needed to successfully coordinate work (Gittell, 2011). Relational coordination (RC) conceptualizes coordination as a set of relationship and communication ties among participants in a work process (Gittell, 2012). The theory contains both relationship and communication dimensions. Relationship dimensions needed for effective coordination are shared goals, shared knowledge, and mutual respect. These dimensions exist between work roles, not individual people (Gittell, 2011). Communication dimensions of relational coordination include communication that is frequent, timely, accurate, and problem-solving (Gittell, 2012). The relationship dimensions reinforce and are reinforced by these types of communication as illustrated in Figure 1.

The theory of relational coordination was developed by Dr. Jody Hoffer Gittell, whose background is in business and organizational management (Relational Coordination Research Collaborative, 2015). The theory emerged from her work in the airline industry, specifically a field study of the flight departure process (Gittell, 2003). Gittell observed differences between communication and relationships among multiple employees at two different airlines (Gittell, 2006). These variations appeared to correlate with work performance and outcomes (Gittell, 2003). Beginning with her observations from this study, Gittell tested and expanded on the

previous work performed by organizational and social science theorists and scholars to develop 
RC (Gittell, 2001, 2006, 2012). Relational coordination has been applied to and tested in healthcare settings (Gittell et al., 2000; Gittell, 2008, 2009; Gittell, Weinburg, Bennett, \& Miller, 2008; Hartgerink et al., 2014; Manski-Nankervis et al., 2014).

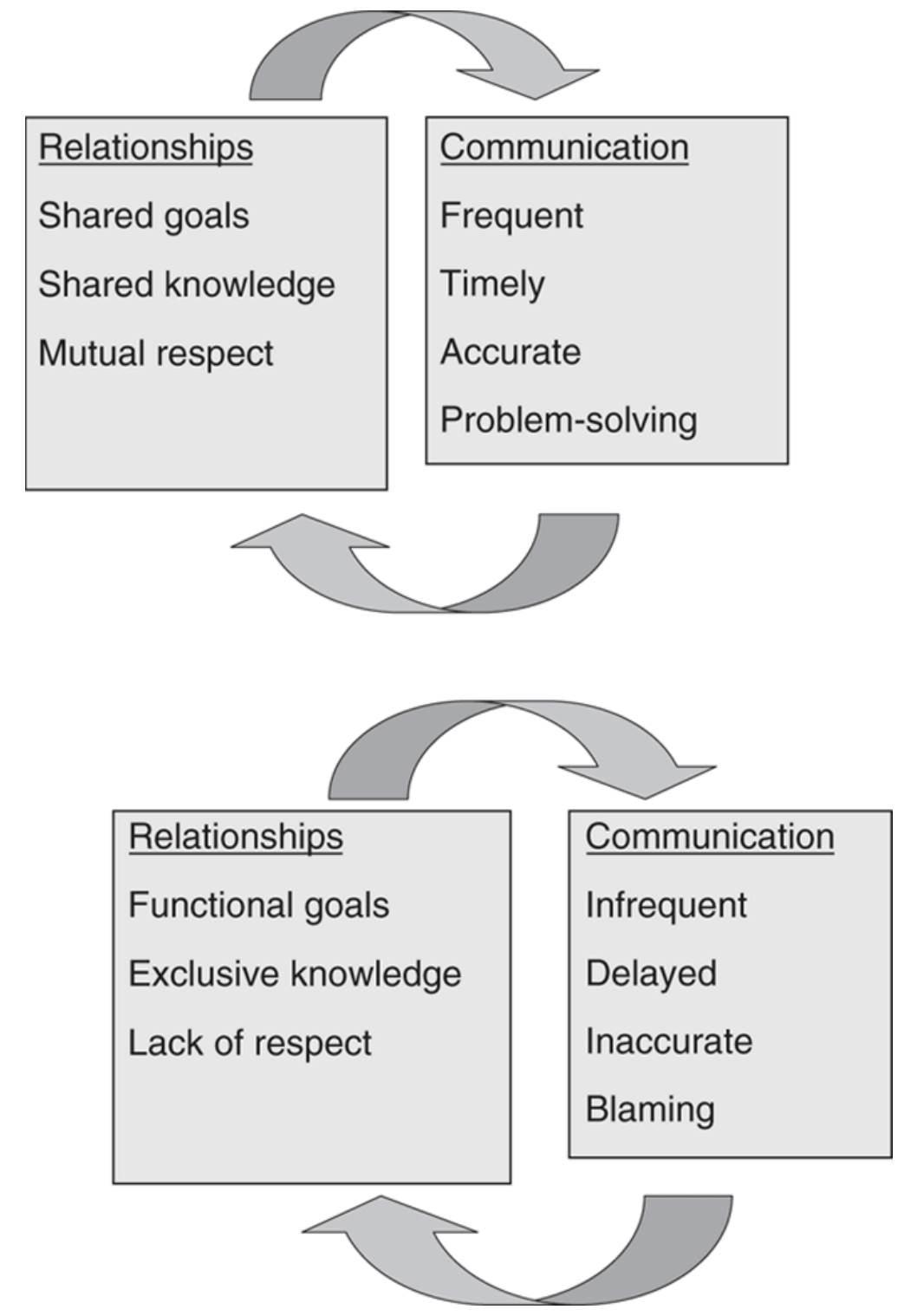

Figure 1. Relational coordination

Gittell, J.H. (2012)

With permission from Jody Hoffer Gittell and Kim Cameron 


\section{Relational Coordination as a Mutually Reinforcing Cycle of Relationships and Communication}

A model of Relational Coordination has been created which describes structures and processes that impact RC as well as how it can affect quality and efficiency outcomes (Gittell, 2012). This model, which is illustrated in Figure 2, allows for the practical application of RC to improve outcomes in healthcare settings.

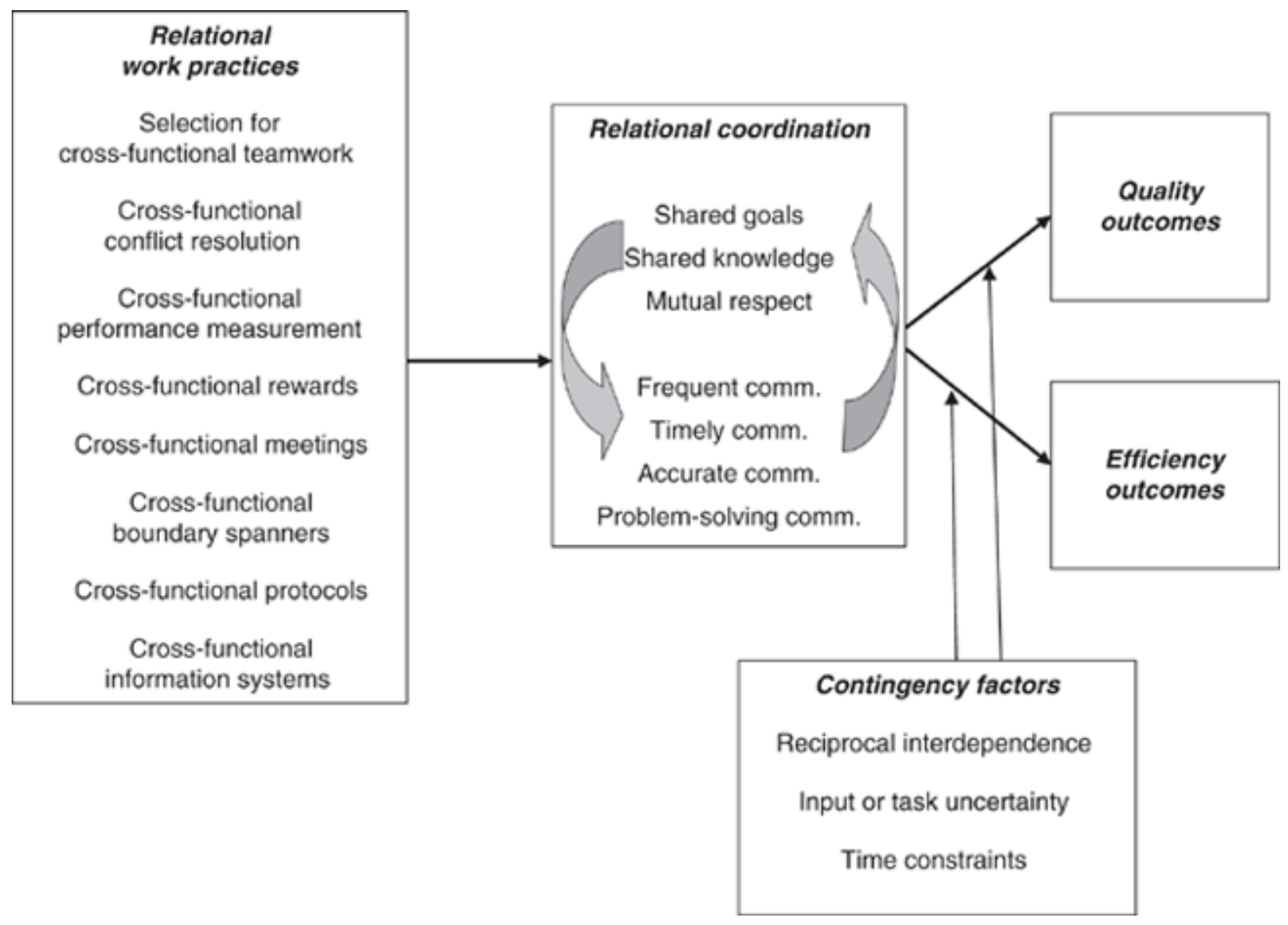

Figure 2. Relational coordination model

Gittell, J.H. (2012)

With permission from Jody Hoffer Gittell and Kim Cameron 


\section{Relational Coordination and ED Physicians}

Relational Coordination is useful in situations with high levels of task interdependence, task uncertainty, and time constraints - conditions that are frequently found in EDs (Gittell, 2006). Relational coordination has been suggested as a useful model for collaboration specifically with physicians because it "does not require redefining roles and transferring authority" (Whitehead, 2007, p. 1014). Functional roles and authority have been cited as barriers to physician engagement in interprofessional collaboration (Flowerdew, Brown, Vincent, et al., 2012; Kilner \& Sheppard, 2010). Factors that have been identified as necessary for physician engagement in collaborative practice or other organizational initiatives include relationship building and development of shared goals (Kaissi, 2014; Kreindler et al., 2012), shared knowledge creation (Morgan et al., 2015), understanding and valuing others' roles or professions (Herbert et al., 2007; Tang et al., 2013; Whitehead, 2007), establishing trust and respect (Herbert et al., 2007; Liebhaber et al., 2009; Tang et al., 2013), and effective communication (Kaissi, 2014; Kreindler et al., 2012; Liebhaber et al., 2009; Lindgren et al., 2013; Morgan et al., 2015; Tang et al., 2013; Whitehead, 2007). These factors all relate to the dimensions of relational coordination.

\section{Relational Coordination and IPCP}

Relational coordination and IPCP are very similar concepts. The idea that the theory of relational coordination can be used to improve IPCP has been suggested by Dr. Gittell and others (Gittell, Godfrey, \& Thistlethwaite, 2013). Interprofessional collaborative practice occurs when multiple healthcare professionals work together. Effective communication and coordination are necessary for this work. Relational coordination addresses the coordination of work processes including the role of communication; therefore, RC is very similar to IPCP and both address problems related to communication and collaboration. The theory describes relational 
components and types of communication necessary to achieve successful coordination.

According to the model of RC, effective RC improves both quality and efficiency outcomes. One of the goals of promoting IPCP is to improve these outcomes. Relational Coordination explains how effective collaboration works, including the role of communication; therefore, it can serve as both a guide and a goal for efforts made to improve interprofessional collaboration.

The use of RC to understand and evaluate what is needed to improve IPCP in EDs does have limitations. The relationship dimensions of RC exist between work roles, not individual people (Gittell, 2011). However, researchers have found that conflicts involving different temperaments and personalities affect communication and teamwork in the ED setting (Cameron et al., 2010; Eisenberg et al., 2006; Flowerdew, Brown, Russ, et al., 2012). The role that individuals play in achieving effective communication and collaboration is not addressed by RC. The impact of stress on this problem is also not included in this theory. The ED environment is stressful and healthcare professionals' reactions to stress have been found to influence how they interact in this setting (Rasmussen et al., 2014; Varjoshani et al., 2014). According to the WHO (2010), IPCP also includes working with patients, families, and communities. Relational coordination only focuses on professionals who are participating in the coordination of tasks and does not address these other participants. These limitations have been acknowledged by the developer of RC (Gittell, 2012). Proposed future development for the theory of RC includes: extending the theory to include personal relationships between unique individuals; building on the social psychological foundations of the theory; and extending RC to include a "broader network of participants" (Gittell, 2012, p. 8). The evaluation of strategies implemented by the participating sites will include their potential effects on improving individual or personal 
conflicts involving physicians. These factors are not addressed by RC but could affect physician engagement in IPCP.

Another potential issue related to the use of RC for this DNP project is the theory's effect on organizational change. The impact of RC on improving outcomes and the need for formal organizational change to sustain RC have been noted (Gittell, 2012). However, the question of whether or not these changes can be successfully implemented without the prior existence of RC has been raised (Gittell, 2012). For example, can an organization successfully implement a new practice in an environment in which co-workers do not already have some level of mutual respect, shared goals, and shared knowledge? If changes in RC must occur prior to changes in formal work structures, then the evaluation of these structures may not be helpful or even possible if the participating organizations do not exhibit any RC. One of the goals of this DNP project is to recognize barriers and needed resources related to strategies implemented to improve physician engagement in IPCP in these sites.

\section{Conclusion}

Negative patient outcomes have been associated with poor communication and collaboration among healthcare professionals in ED settings. Interprofessional collaborative practice has been recommended as a solution to this problem. In order to benefit from this solution, organizations must be able to successfully engage physicians in strategies to promote IPCP in their settings. Improved IPCP with physicians to provide high quality patient care in rural EDs can be guided by RC as it explains what is needed to accomplish effective work coordination between healthcare professionals. Relational coordination has been shown to be effective for improving outcomes in other healthcare settings and industries. 


\section{CHAPTER 4: METHODOLOGY Project Design}

This DNP project is an exploratory multi-case study of four rural North Carolina EDs participating in the Shaping Systems quality improvement collaborative project designed to improve interprofessional collaborative practice. Qualitative data were collected through project participation, observation, and scheduled interviews and used to examine and report on physician engagement in interprofessional collaborative practice in these settings. Data collected for the Shaping Systems project was also used to provide additional context related to physicians at each of the participating sites.

\section{Participants}

For the Shaping Systems project, partner hospitals formed teams that participated in project activities and learning collaborative meetings. In addition, each hospital designated a site coordinator for the project. The site coordinators from each site were nurse leaders in the EDs, such as ED directors, ED nurse managers, or nurse educators. Each hospital's project team members and site coordinators were the primary subjects or informants for this DNP project.

The hospitals formed these teams and chose coordinators based on their own assessment and needs for creating IPCP in their EDs; therefore, participants varied across the four settings. Hospitals were encouraged to create teams that consisted of ED nurses and physicians as well as other professionals, units, departments and roles that they felt were most important to coordinate quality ED care in their setting. The participants from each hospital also varied across Shaping Systems quality improvement collaborative events and activities. Participants for this DNP 
project were a convenience sample based on availability of team members, hospital staff, and site coordinators at different data collection points.

\section{Settings}

\section{Shaping Systems Grant Project}

The primary setting for this DNP project was the HRSA-sponsored project, "Shaping Systems to Promote Desired Outcomes: Interprofessional Collaborative Practice in Rural North Carolina EDs." The project's principal investigator was Dr. Donna Havens at the University of North Carolina at Chapel Hill School of Nursing. This project was designed to implement a four-hospital quality improvement collaborative to promote nursing's capacity to lead development of and the competency to practice in IPCP environments in the ED setting. The quality improvement collaborative provided participating hospitals with learning opportunities and resources for practicing and leading in IPCP environments. Hospitals could adapt what they learned and implement strategies they felt worked in their local context.

Activity components of the project included quarterly learning collaborative meetings and site visits. Each hospital sent a team to attend these meetings. Hospital teams were exposed to different resources, skills, and strategies as well as given opportunities to share their challenges and successful approaches for improving IPCP. Members of the Shaping Systems team made site visits that were scheduled according to need and availability.

The Shaping Systems project was developed to improve patient and staff outcomes in rural EDs in North Carolina. Rural hospitals face unique challenges related to limited access to professional education, isolated geographic locations, lower compensation and fewer benefits, and other socioeconomic hardships. The four hospitals participating in this project were chosen based on their rural locations and service to residents in a Health Professional Shortage Area and Medically Underserved Area. 


\section{Chatham Hospital}

Chatham Hospital is a 25-bed, not-for-profit, critical access hospital in Siler City, NC. Critical access hospitals are part of a federal program to improve access to rural health care and reduce rural hospital closures. The emergency department has 10 beds and reported 14,449 ED visits in 2013. This hospital is part of the University of North Carolina (UNC) Health Care system. In the emergency department, UNC Health Care physicians are contracted to work alongside Chatham Hospital nurse practitioners, physician's assistants, and nursing staff.

\section{Pardee Hospital}

Pardee Hospital is a 222-bed, not-for-profit hospital located in Hendersonville, NC. This hospital is also part of the UNC Health Care system. The emergency department has 32 beds and reported 31, 800 ED visits in 2013. In 2015, Pardee announced that the Hendersonville Emergency Consultants Group, who provided physicians and midlevel providers for Pardee's Emergency Department, became directly employed by Pardee under the name Pardee Emergency Physicians.

\section{Southeastern Medical Center}

Southeastern is located in Lumberton, NC and licensed for 452 beds. The hospital is part of the not-for-profit Southeastern Health system which has been designated as a Magnet ${ }^{\circledR}$ organization. The emergency department has 63 beds and reported 74,650 ED visits in 2013. The hospital has contracted with Emergency Medicine Associates, P.A., P.C. to provide physicians and midlevel providers for their emergency department. Medical residents, including four in emergency medicine from Campbell University, began working for Southeastern in 2015. 


\section{Transylvania Regional Hospital}

Transylvania is a 52-bed, not-for-profit, critical access hospital in Brevard, NC. This hospital is part of the Mission Health system. The emergency department has 10 beds and reported 14,077 ED visits in 2013. In the past, the hospital has contracted with a private company to provide physicians for their emergency department; however, their ED physicians are now employees of the Mission Health system.

\section{Data Collection}

Data for this DNP project were obtained by the investigator, a DNP student in the School of Nursing at the University of North Carolina at Chapel Hill during the second and third years of the three-year Shaping Systems project. The investigator collected observational data at regularly scheduled collaborative learning sessions as well as other events, such as site visits, coordinated by Shaping Systems project team members. She collected interview data as part of data collection phone interviews conducted for the larger project. Institutional Review Board (IRB) approval was obtained for the Shaping Systems project from UNC-Chapel Hill and each participating hospital and renewed annually. Additional IRB approval for this DNP project was also obtained.

\section{Observations}

Observations were conducted during investigator participation in Shaping Systems events, such as regularly scheduled learning collaborative sessions and site visits. Collaborative learning sessions occurred over a two-day period and were primarily held in a central location, Greensboro, North Carolina; however, the session held in August 2016 took place at one of the participating hospitals. The dates for these meetings were announced 4-6 months ahead of time. The DNP student investigator attended five of the learning collaborative sessions for a total of nine days. The student investigator participated in three site visits, two that were arranged by 
"Shaping Systems" team members according to project and site needs and one site visit that she arranged with one of the sites' ED director. The data collection for these events included notetaking when discussions were related to physician participation in either the Shaping Systems project or any hospital-related practices involving interprofessional collaboration. Observation notes were typed up using Microsoft Word and stored on a password-protected computer.

\section{Interviews}

Data were also gathered during planned telephone interviews that a Shaping Systems team member conducted annually with the site coordinator at each hospital. Interviews focused on project-related changes that had been made at the sites as well as barriers encountered. The DNP student investigator participated in interviews conducted at the end of Shaping Systems years one and two. Interview responses from both years were used to gather qualitative data for this DNP project. As part of the interviews conducted at the end of year two, the DNP student investigator asked additional open-ended questions related specifically to physician engagement.

Participants requested a copy of interview questions prior to the scheduled telephone interview. Participants acknowledged needing more time to think about the questions and consult with others if needed to find answers. This request was granted and a copy of the interview guide for this DNP project was given to participants prior to the interviews. This strategy was appropriate as participants were describing what they were doing in their settings and it enabled participants to provide more complete information. Conducting the open-ended telephone interviews allowed for follow-up questions and clarification as needed.

Interviews were recorded and transcribed. Copies of the transcriptions were given to the DNP student investigator and stored on a password-protected computer. 


\section{Interview Guide}

The telephone interview conducted at the end of the second year of the Shaping Systems project included questions related specifically to physician engagement. These questions were developed by the DNP student investigator. The interview guide for these questions can be found in Appendix A. Questions focused on facilitators, barriers, needed resources, and successful strategies related to improving physician engagement in both the Shaping Systems project and interprofessional collaboration efforts at the participating sites. These questions were added to ensure that data on physician engagement were collected from each site regardless of whether it was discussed elsewhere during the interview.

\section{Contextual Data}

Information about the hospital sites size and number of ED visits was obtained from the Shaping Systems project team. Additional contextual data were collected by the DNP student investigator during participation in the Shaping Systems learning collaborative sessions. These data included information about the relative number of physicians at each site and their employment or contractual relationships with the organizations as well as information related to ED leadership turnover. Information related to participant attendance in Shaping Systems activities was obtained from the project manager. These data included attendance at learning collaborative meetings as well as on-site learning activities. Site team coordinators were asked to provide the profession and role of each team member that had participated in Shaping Systems activities that were used for data collection for this project. Information about participants at site visits was obtained by the student investigator as part of the field notes. Interview participants were asked for this information during the interviews. 


\section{Data Analysis}

Transcribed observation notes and interview responses were read for content and excerpts related to physicians or physician engagement were selected. These excerpts were organized by hospital and copied into separate Microsoft Word documents. Excerpts were then reviewed using content analysis and deductive coding (Miles, Huberman, \& Saldana, 2014). "Contextual factors" and "strategies" were used as codes for organizing data in the first cycle of coding based on the project's research question (Miles et al., 2014). Information identified as "contextual factors" was reviewed and noted to be "barriers" or "facilitators" to physician engagement.

Cross-case analysis was then used to explore similarities and differences across the four participating ED sites as well as how local conditions affected their strategies and outcomes (Miles et al., 2014). Data excerpts addressing similar concepts or topics were combined into Microsoft Word tables after being color-coded according to colors assigned to each site. These tables served as conceptually clustered matrices (Miles et al., 2014). The data within these tables were then analyzed and assigned sub-codes using inductive coding. These sub-codes provided more detail within the broader categories of "contextual factors" and "strategies" (Miles et al., 2014). The sub-codes assigned were descriptive labels comprised of words or short phrases. The resulting tables allowed the investigator to describe the contextual factors and strategies used by the different ED sites based on coded and sub-coded categories or themes. The colorcoded table entries enabled the investigator to identify the number of sites that addressed or identified each category, while also preserving the confidentiality of each site.

\section{Data Validation}

Qualitative data coding was reviewed by a co-reader with expertise in qualitative data analysis. The co-reader was a Shaping Systems team member who conducted the qualitative interviews for the larger project and was therefore familiar with the participating sites. The DNP 
student investigator and co-reader worked together to reach consensus on the final data categories. The DNP student investigator presented the findings to the site team members who were present for the Shaping Systems learning collaborative in January 2017. Team members from all of the sites expressed their agreement with the final categories and examples presented. This presentation served as "participant checking" to ensure that the findings represented the site teams' meanings, and perspectives were accurately represented (Tong, Sainsbury, \& Craig, 2007). During the presentation, site team members discussed the issue of physician engagement and provided additional information which was subsequently added to the existing data. The new data did not require the addition of any new categories or codes. 


\section{CHAPTER 5: RESULTS}

\section{Sample}

The composition and number of members that participated in events used for data collection differed for each of the four ED site teams. The green hospital had the largest number of team members $(n=17)$ participate in events where data were collected. The orange hospital had 13 team members participate in study events. This hospital had the largest number of events related to this project. The red hospital had a total of 10 participants, including a few participants that were not members of their Shaping Systems team at the physician shadowing site visit. The hospital with the fewest participating team members was the blue hospital $(n=6)$. While this site did not have as many participants, they had a very consistent core team that attended all the learning collaborative sessions. A complete list of participants in data collection events is found by type of event in Tables 1,2 , and 3. 
Table 1. Participants in the 5 learning collaboratives associated with this project

\begin{tabular}{|c|c|c|}
\hline Hospital & Professions & Job titles \\
\hline Green & $\begin{array}{l}15 \text { Registered Nurses } \\
1 \text { Respiratory Therapist } \\
1 \text { Other }\end{array}$ & $\begin{array}{l}\text { - Manager of Emergency Services and } \\
\text { - Elinical Operations } \\
\text { - ED Nurse Manager } \\
\text { - ED Nurse x } 5 \\
\text { - ED Nursing Unit Supervisor } \\
\text { - ED Charge Nurse } \\
\text { - ED Preceptor Nurse } \\
\text { - ICU Nursing Unit Supervisor } \\
\text { - ICU Nurse } \\
\text { - Medical Surgical Nursing Unit } \\
\text { - Supervisor } \\
\text { - Director of Clinical Operations } \\
\text { - Rerformance Improvement consultant } \\
\text { Respiratory Therapist }\end{array}$ \\
\hline Blue & 6 Registered Nurses & $\begin{array}{l}\text { - ED Nurse Manager } \\
\text { - ED Director } \\
\text { - Clinical Nursing Coordinator } \\
\text { - Director of PATHS (Psychiatric \& } \\
\text { Addictions Therapeutic Healing } \\
\text { Services) } \\
\text { - PATHS Nurse }\end{array}$ \\
\hline Red & $\begin{array}{l}5 \text { Registered Nurses } \\
2 \text { Physicians } \\
1 \text { Respiratory } \\
\text { Therapist } \\
2 \text { Other }\end{array}$ & $\begin{array}{l}\text { - ED Director } \\
\text { - ED Nurse x } 2 \\
\text { - ED Co-Medical Director } \\
\text { - ED Co-Medical Director } \\
\text { - Nurse Educator x } 2 \\
\text { - Respiratory Therapy Director } \\
\text { - Patient Registration/Access Director } \\
\text { - Lab Director }\end{array}$ \\
\hline
\end{tabular}




$\begin{array}{ll}\text { Orange } 10 \text { Registered Nurses } & \text { - Interim ED Director x } 2 \\ 1 \text { Physician } & \text { - ED Director } \\ & \text { - ED Nurse Coordinator } \\ & \text { - ED Nurse Manager } \\ & \text { - ED Associate Medical Director } \\ & \text { - Critical Care Transport Coordinator } \\ & \text { - Chief Nursing Officer } \\ & \text { - Clinical Applications Analyst } \\ & \text { - Director of Clinical Services for } \\ & \text { Physician Contract Company } \\ & \text { - Director of Care Management } \\ & \text { - Director of Clinical Services } \\ & \text { - Director of Behavioral Health } \\ & \text { - Chief Process Design Officer }\end{array}$


Table 2. Interview participants

\begin{tabular}{|c|c|c|c|}
\hline Hospital & Interview Year & Professions & Job Titles \\
\hline \multirow[t]{2}{*}{ Green } & Year 1 (2015) & Registered Nurse & $\begin{array}{l}\text { - Manager of } \\
\text { Emergency Services } \\
\text { and Clinical } \\
\text { Operations }\end{array}$ \\
\hline & Year 2 (2016) & 3 Registered Nurses & $\begin{array}{l}\text { - ED Manager } \\
\text { - ED Nurse } \\
\text { - Manager of Clinical } \\
\text { Operations }\end{array}$ \\
\hline \multirow[t]{2}{*}{ Blue } & Year 1 (2015) & 2 Registered Nurses & $\begin{array}{l}\text { - ED Nurse Manager* } \\
\text { - Interim ED Director }\end{array}$ \\
\hline & Year 2 (2016) & Registered Nurse & - ED Nurse Manager* \\
\hline \multirow[t]{2}{*}{ Red } & Year 1 (2015) & Registered Nurse & $\begin{array}{l}\text { - Hospital Nurse } \\
\text { Educator }\end{array}$ \\
\hline & Year 2 (2016) & Registered Nurse & - ED director \\
\hline \multirow[t]{2}{*}{ Orange } & Year 1 (2015) & 3 Registered Nurses & $\begin{array}{l}\text { - Interim ED Director } \\
\text { - ED Nurse Manager } \\
\text { - ED Nurse } \\
\text { Coordinator* }\end{array}$ \\
\hline & Year 2 (2016) & 2 Registered Nurses & $\begin{array}{l}\text { - ED Nurse Manager } \\
\text { • ED Nurse } \\
\text { Coordinator* }\end{array}$ \\
\hline
\end{tabular}

*=Same person participated in interviews both years 
Table 3. Site visit participants

\begin{tabular}{|c|c|c|}
\hline Hospital & Type of Site Visit & Participants \\
\hline \multirow[t]{6}{*}{ Orange } & $\begin{array}{l}\text { Observation of Friday Night } \\
\text { in the ER simulation }\end{array}$ & $\begin{array}{l}\text { - Managers from multiple } \\
\text { patient units and } \\
\text { departments }\end{array}$ \\
\hline & & $\begin{array}{l}\text { - ED medical director } \\
\text { - ED nurse coordinator } \\
\text { - ED nurse manager }\end{array}$ \\
\hline & & - Physician Assistant \\
\hline & $\begin{array}{l}\text { Physician recruitment visit } \\
\text { with Shaping Systems } \\
\text { physician lead }\end{array}$ & $\begin{array}{l}\text { - ED nurse coordinator } \\
\text { - Interim ED director } \\
\text { - Future ED nurse manager }\end{array}$ \\
\hline & & $\begin{array}{l}\text { - Chief Nursing Officer } \\
\text { - Chief Operating Officer }\end{array}$ \\
\hline & & $\begin{array}{l}\text { - Physician Assistant } \\
\text { - Nurse Practitioner } \\
\text { - } 4 \text { Physicians }\end{array}$ \\
\hline Red & ED physician shadowing & $\begin{array}{l}\text { - ED director } \\
\text { - Physician } \\
\text { - Resident Physician } \\
\text { - Physician Assistant } \\
\text { - Scribe }\end{array}$ \\
\hline
\end{tabular}




\section{Findings on Physician Engagement in Interprofessional Collaborative Practice Activities}

Participants described physician engagement (or lack of engagement) in three types of ICPC activities: Shaping Systems events, everyday work, and process or quality improvement initiatives. Shaping Systems events included both quarterly learning collaboratives held off-site and on-site events sponsored by the collaborative project. Everyday work included activities such as meetings, huddles, and routine patient care. Process or quality improvement initiatives, including Lean projects or Rapid Improvement Events, were frequently mentioned opportunities for IPCP. The qualitative data collected for this project related to physician engagement in all three of these types of IPCP activities or efforts and included both contextual factors and strategies that influenced physician engagement.

\section{Contextual Factors Influencing Physician Engagement}

Site team members from the four EDs described seven categories of contextual factors that influenced their ability to engage physicians in IPCP activities: employment arrangements, scheduling, competing priorities, leadership, work environment, resources, and knowledge. These contextual factors were most often identified as barriers to physician participation. However, in some cases participants described factors that facilitated physician involvement.

Employment arrangements. All four site teams mentioned issues related to the physicians' employment arrangements. Employment arrangements varied among sites with some physicians provided by outside contract agencies or organizations and others working as employees of the hospital or healthcare system. Employment arrangements and physician contracts determined how physicians' time was allotted, compensation, accountability, as well as influenced investment in the organization. 
Time allotment \& compensation. Three site teams indicated that employment contracts designate separate administrative and clinical time for physicians. According to team members, physicians use administrative time to participate in IPCP efforts and this time is limited. One ED nursing coordinator stated that their medical director "has too much clinical work and is not given enough time for meetings or administrative work." Lack of compensation is a related barrier identified by three site teams. One team member claimed, "they will not attend meetings without being paid and are not as likely to come to voluntary meetings." Physicians are given a limited amount of administrative time for all non-clinical activities which also includes education and vacation. Site teams discussed lack of compensation as a reason for the absence of physician participation in Shaping Systems collaborative meetings, rapid improvement events, as well as routine meetings.

Accountability. Team members from three sites mentioned ways in which accountability affected physician engagement in IPCP efforts. Accountability includes physicians' obligation to participate in hospital committees, projects, or events, as well as their responsibility for quality or process outcomes. Two sites discussed physicians' responsibility or accountability for patient care quality or process metrics in relation to physician engagement in IPCP endeavors. An ED director from one of the sites noted the lack of options for getting physicians to participate and stated that there are "no repercussions for physicians not attending meetings." He also asserted that "physicians are not held responsible for metrics" which contributes to the challenge of getting them involved in improvement efforts. In contrast, a third site team stated that some metrics have been written into their physician contracts. Reimbursement from the physician contract company is tied to patient care metrics, which they believe has increased physician interest in working with others on some improvement projects. 
Investment. Physician investment in or loyalty to the hospital was discussed as a factor related to IPCP at three sites. Team members questioned their physicians' level of support for the hospital and its staff. A physician at one site acknowledged that ED physicians are often uncertain of their loyalty to the hospital versus their contract company. One site team member at a different hospital mentioned that physicians who are temporary or part-time are "just interested in getting their shifts done" and not willing to put in the extra time for IPCP activities. A physician who was filling in temporarily at the same site admitted that his temporary status deterred him from wanting to engage in IPCP efforts. The third site team reported mixed views of physician loyalty. One team member remarked that their medical director was much more involved with the hospital and community than other ED physicians, including serving on the hospital foundation and helping with fundraising. She felt that although he is also a contract employee, he exhibited more involvement and buy-in than other ED physicians for improving patient care and processes such as ED throughput times. A member of the same site team said "I feel like physicians are still not vested in the hospital. They are contract employees in their mind. They are also not locally vested and don't live in the community." Three of the sites acknowledged that the contract employment status of their physicians and their rural location resulted in many physicians commuting large distances to work in their EDs. At one site, an ED nurse expressed disappointment that their ED physicians “don't invest in the hospitals where they work."

Scheduling. All four site teams discussed challenges related to scheduling and physician engagement in IPCP. One nurse manager asserted that "scheduling is honestly probably the biggest challenge" to physician participation in the Shaping Systems project and other 
interprofessional efforts at her hospital. Challenges included pulling physicians from patient care, aligning physician and nurses' schedules, and location.

Pulling physicians from patient care. The nature of physicians' work in EDs creates obstacles to their ability to participate in IPCP activities that take place outside of everyday clinical responsibilities. The need to ensure adequate staffing for patient care was identified by three sites as a challenge to getting physicians to attend Shaping Systems collaborative meetings or interprofessional education events. One ED director noted that having only a small number of physicians on staff means that "someone has just worked or is currently working" at all times. Similarly, a participant from one site team reported that shift work makes it difficult for physicians to attend meetings. According to this team member, physicians working night shift exhibit the lowest levels of buy-in for IPCP activities. Two site teams reported difficulty scheduling meetings over the summer months due to conflicts with vacations and patient care staffing. This stemmed from physicians being required to use administrative time, not clinical time, for vacations. As a result, physicians have less time for meetings or other IPCP activities during months when they are more likely to take time off.

Aligning schedules. Members of two site teams discussed obstacles related to nurse schedules and availability. Nursing staff shortages and the need to ensure enough nurses for patient care were reported as impediments to nurses attending events with physicians, such as resident training classes. This site also noted that the focus for educational events was often on fitting nurses' schedules, which did not align with physicians' schedules.

Location. Team members from two sites mentioned that the location in which IPCP activities or events are scheduled influences physician participation. One site noted that the large distance from their hospitals to quarterly Shaping Systems learning collaboratives was a 
challenge to getting physicians to participate as the distance required physicians to schedule more time off to participate. Similarly, a member from another site reported that their physicians do not live close to the hospital, which creates challenges to scheduling onsite IPCP activities because physicians do not want to travel to the hospital when they are not working.

Competing priorities. Every site team discussed challenges related to the high number of competing priorities for physician time and attention. Competing priorities arise from physicians' and staff's multiple commitments as well as the different responsibilities of physician specialties. All site teams recognized that physicians are frequently dealing with many different responsibilities and a limited amount of time.

Multiple commitments. Each of the four site teams addressed the challenge of physicians being requested to be involved in too many different activities. All teams described their ED and/or hospital's involvement in many different improvement projects, initiatives, and collaboratives. Examples of the multiple competing commitments and issues discussed by all four ED site teams are listed in Table 4. 
Table 4. Projects, initiatives, collaboratives, issues discussed by ED site teams

\begin{tabular}{ll} 
Healthcare system value program & ICU rapid admit process \\
State-wide Transitions of Care collaborative & Language of Caring classes \\
New EHR implementation & TeamSTEPPS \\
Lean projects & Stand out program \\
Rapid Improvement events & Purposeful hourly rounding \\
ED patient throughput task force & Bedside shift report \\
Leaving Without Being Seen (LWBS) rates & Leader rounding \\
Healthcare system caring program & Patient satisfaction/experience scores \\
ED functional team & County collaboration for psychiatric patients \\
Stroke center designation & Performance pulse \\
Bed Ahead process & Patient advisory committee \\
ED renovations & Transfer review team \\
Discharge implementation plan & Surge plan - Code Surge \\
Staff retention & Trauma protocol \\
Length of stay (LOS) metrics & Hospital throughput - Teletracking \\
Accountability coaching & Psych holding - implementation of safe unit \\
Increased volumes & Paramedic job duties review \\
Psych education & Room standardization \\
New MD staffing model & Bridge orders \\
Decreasing sepsis mortality & Fast track \\
\hline
\end{tabular}

Two of the sites were involved in several initiatives that were sponsored or required as a result of belonging to a larger healthcare system. For example, one hospital spent over a year preparing for and initiating the use of a new electronic health record system. Another team linked the number of competing projects or efforts to the limited amount of administrative time physicians were given for events like IP meetings or educational events. A participant from another site team noted the difficulty of getting physicians and staff involved due to their staffing 
and educational commitments as well as participation on other committees. One physician who was interested in attending a Shaping Systems collaborative meeting was unable to due to a conflict with another conference.

Different responsibilities. Site teams frequently discussed the need to collaborate with physicians outside of the ED. For example, hospitalists and intensivists play a role in the care of patients being admitted from the ED. Two of the hospital site teams brought up challenges created by the different priorities of non-ED physician specialties. One team member noted that it was difficult to coordinate intensivists with hospitalists. The other team spoke specifically about competing priorities for hospitalists. One member said that "the hospitalists are pulled in so many areas at times" and "they think that other things are more important and they have different priorities." One site also discussed the challenge of working with physicians that have joint academic appointments and clinic responsibilities in addition to their ED work. These physicians work only part time or periodically in the ED and spend time seeing patients in an outpatient primary care setting as well as teaching at the medical school.

Leadership. The impact of leadership on physician participation in IPCP efforts was mentioned by all four site teams. All teams recognized the need for leadership support to successfully engage physicians in IPCP activities. The significance of leadership was illustrated by the level of support given by leaders and absence and turnover of leaders. Leadership at all levels was discussed, including physician leadership, nursing leadership, hospital administration, and ED level leadership.

Level of support. Participants from three of the sites discussed examples of leadership support that promoted physician engagement. One hospital team reported that they receive help from their hospital leadership in that "administration sets the expectation" that participation in 
IPCP will occur. Two hospital executives from a different site described their support of IPCP and improved collaboration between and among physicians and other staff to improve patient care. Another site described the positive impact of their medical director's communication with physicians to encourage participation in meetings. While several sites described good collaboration and support for IPCP from leadership, two of the sites expressed concern that these efforts were not diffusing down to the lower levels of physicians and staff. One site team member suggested "buy-in is not getting down to the grassroots provider levels." Similarly, a different site team participant noted that they "need to include frontline staff in the collaboration - not just between physicians and leadership."

Absence and turnover. Two of the site teams reported that recent turnover in multiple leadership roles had created a barrier to physician engagement in IPCP activities. One team reported turnover in several ED hospital leadership positions as well as physician leadership turning over several times in a small amount of time. Another site team noted that the absence of a Chief Medical Officer has resulted in lower levels of physician engagement because no one was overseeing their activities or holding them accountable.

Work environment. Each of the site teams discussed or displayed aspects of the ED work environment that affected physician engagement in IPCP. Characteristics of the work environment included aspects of the physical layout of the hospital units, workflow, electronic health record systems, and culture or climate. The work environment influences physicians' ability and willingness to work with other professionals.

Physical layout. A site visit to one ED revealed that the central workstation was divided into two distinct areas separated by a medication workspace. The nurses worked in one area of the workstation while the physicians spent their time in the other area. Minimal interaction 
between nurses and physicians was noted. This workspace design was also mentioned by the ED director as a deterrent to collaboration between nurses and physicians. Two other site teams reported examples of workspace facilitating IPCP. One ED director mentioned that having an office right next door to the medical director has improved their working relationship by providing frequent interaction. They stated that this office arrangement "helps with traction and it is good to know you have a physician champion on your side." Another site team pointed out that physician participation in huddles was the result of physicians being at the nursing station when they occur. According to one team member, the physicians "have nowhere else to run to except their breakroom, so they are there listening passively and actively, kind of forced to participate because we're standing around them."

Work flow. Two workflow issues that affect IPCP with physicians were found at one participating ED. During a site visit, the nurse and physician workflows were not observed to include collaboration during patient care. The physician and the nurses saw patients separately and only interacted briefly when the physician needed to give the nurse a new order. The physician being observed commented that "I let the nurses do their part and then I go in to see the patient when they are done." This issue was also mentioned by a second site team whose member noted that they have similar issues with physicians and nurses not going into patient rooms together. The team member mentioned that they have been working to change their workflow process to allow for these clinicians to see patients simultaneously. At the first site, one team member noted that having different admission processes for day and night shifts affects interactions between hospitalists and ED physicians. The team suggested that collaboration is better during the night shift due to the admission process used at night. 
Electronic health record systems. Patient health records or charts can serve as a means for collaboration and communication among healthcare providers. All of the site teams brought up issues related to their electronic health record (EHR) systems that impact physician engagement in IPCP. One site noted that currently their physicians chart in a different system than the rest of the staff. Working in different systems limits communication and prohibits staff and physicians from seeing the work done by others. Another site obtained a new EHR system in the last year. Site team members reported that during training on the new system, staff and physicians were only instructed on their own charting workflow and not how their charting affected or could be viewed by others. In this case, the physicians and staff work in the same system but may not know how to collaborate with others in this new system. The ED director at this site noted they had to develop "workarounds" to establish a process for communication between physicians and nurses in their EHR system. A physician from another site commented on her inability to see important aspects of the chart, such as the medication administration record and medication list which had been completed by the nursing staff and pharmacy. She noted that she had to print out discharge information for the patient in order to see these sections of the chart. The fourth site team remarked that their EHR system limits interactions between physician specialties based on what can be done by the different roles within the EHR system. The same site team also commented that the EHR provides some opportunities for better collaboration. In the case of their boarder patients, required documentation in the EHR by different healthcare professionals forces collaboration and clarifies who is responsible for different aspects of care. 
Culture or climate. The effect of the overall culture and climate in the ED work environment was discussed by one site team. Two members of this site team addressed "culture and physicians not wanting to change" as their biggest challenge to improving physician engagement in IPCP. This team also addressed the impact of overall staff morale on IPCP. They noted improvement in physician IPCP, as staff morale in the ED has improved over the last year. An ED physician from this site also noted that the culture at the hospital is changing as "the residency program has attracted physicians with better attitudes toward change and collaboration." An ED physician from a different site noted that she gets job satisfaction from providing direct patient care and teaching. She is less interested in working on IPCP activities such as improvement initiatives and going to meetings. This hospital site team noted that many of their physicians were hired to teach at the nearby medical school and that there is a tension between teaching and working on improvement efforts in this site's ED. All of the hospital teams discussed lack of trust and climate between hospitalists and ED physicians as a barrier to IPCP between physicians. The teams all reported a "lack of trust" or "resistance" between hospitalists and ED physicians that impeded efforts to improve patient care processes such as admissions.

Resources. Three of the site teams brought up different resources that served as either facilitators or barriers to physician engagement in IPCP. Lack of needed resources in the ED was cited as an impediment to IPCP with physicians while outside resources were discussed as opportunities to engage physicians in IPCP.

Lack of needed resources. Two site teams identified lack of key personnel as barriers to IPCP opportunities for physicians. One ED director stated that he was unable to share data with physicians because the ED did not have a staff member that could run the data reports he needed 
from the EHR system. Another site team noted that they did not have an ED nurse educator to support learning activities for physicians and other staff. They claimed that an educator dedicated to the ED would provide opportunities for interprofessional education, which would in turn support more IPCP.

Outside resources. Three of the site teams discussed resources or opportunities outside of their EDs that could be used to engage physicians in IPCP. For one site, the establishment of a residency program at their hospital has created such a resource. The site team members have discussed leveraging the residency program to provide IPCP opportunities, such as the inclusion of residents in interprofessional huddles and making residency-training events open to nurses. A physician at this site also identified interprofessional education opportunities in their rural setting. Opportunities included county disaster response and joint paramedic training. This site has also found that mandatory hospital-wide training classes, such as a language of caring class and TeamSTEPPS, have provided opportunities for interprofessional education which supports IPCP. Another ED team pointed out that as a small hospital, all committees are hospital-wide and interdisciplinary. These committees are an existing structure for IPCP that can include physicians. Two site teams identified the ability to participate in collaboratives, such as Shaping Systems, and other outside initiatives as a facilitator to including physicians in IPCP. Even if physicians did not participate, one hospital team noted that "being able to talk to others, especially from other hospitals to see how they solve issues" was an opportunity to bring new ideas and solutions back to the physicians in their hospitals. Participation in a Shaping Systems collaborative meeting was found to be very engaging for a physician at one site. After talking to other site teams about similar issues she said she was "fired up" to go back and work with her interprofessional team to make improvements in their ED. 
Knowledge. All four of the participating site teams discussed examples that illustrated how information positively or negatively affected physician engagement in IPCP. Physicians' knowledge of patient care issues faced by the ED as well as site team members' knowledge of physician employment arrangements were shown to influence efforts to involve physicians in IPCP. Better knowledge about these topics led to increased understanding and ability to get physicians to participate while the lack of knowledge appeared to be a barrier.

Physicians' knowledge of patient care issues. Three of the site teams discussed the impact of physicians' awareness of the problems they faced in the ED. One site team member admitted that they did not know what information about ED issues or data was shared with physicians on other units. Similarly, another site team stated that often their medical director did not share data with physicians after it was shared with him. In both examples, physicians who are needed to help work on improving patient care issues in the ED are likely not fully aware of the problems. A team member from a different site suggested that physicians need more information or data about their influence on common ED challenges. "I don't think each individual physician realizes the impact they personally have on the ED throughput." Conversely, two of these site teams acknowledged that overall physicians were "beginning to feel the frustration with how the ED was working" and know that "changes need to take place." At the last learning collaborative, the nurse coordinator from one site relayed an example of one physician recognizing a patient flow problem and taking the initiative to pull patients herself to the fast track area which was the only place available to place patients at that time. Because physicians were gaining this general knowledge, these teams felt that they were more motivated to help solve problems and were slowly becoming more involved. 
Site team members' knowledge of physician employment arrangements. Members of two site teams admitted they were not sure how their physicians' employment arrangements impacted their participation in IPCP activities. One ED director stated "I don't know the physician structure side. What are they required to do in terms of participation in committees or meetings, etc. Do they get education or administrative time? Are they reimbursed?" Another team had concluded that physicians were no longer attending some interprofessional meetings because they had become employees of the hospital. When asked for more details, they acknowledged that they were uncertain about how changes in employment status impacted physician participation. The team members had just assumed there was a link but did not know if the change in employment status had affected their reimbursement or requirements. In both cases, team members conceded that having more information about physicians' employment arrangements would aid in planning and including them in IPCP efforts. At the last learning collaborative, the ED director reported that he had inquired more about their physicians' contractual obligations to the hospital. He stated that the knowledge he gained has allowed him to better understand the physicians' perspective and needs. Only $40 \%$ of the physicians' time is dedicated to their hospital while the other $60 \%$ is spent at the larger academic medical center affiliated with the same healthcare system. This information has made him more aware of the need to help physicians become more comfortable with the processes at his hospital.

\section{Strategies}

All four of the ED site teams shared strategies they have used to engage physicians in their IPCP efforts. These strategies fell into four general categories: structural, logistical, interpersonal, and quality improvement related. As detailed below, specific strategies were found to be successful, unsuccessful, or resulted in mixed success in improving physician involvement in IPCP. 
Structural - Involving physicians in structures already in place at the hospital. Each of the site teams identified strategies that included physicians in events, activities, or procedures that were in place in their settings. Existing arrangements used to engage physicians in IPCP efforts included meetings, huddles, education, and policies or processes. The ED teams used these strategies to try to hardwire physician engagement into their systems.

Meetings. All four site teams used meetings to bring physicians together with other staff or leaders. Differences were found in who was asked to participate, what type of meeting was involved, and how often meetings occurred. In some cases, meetings were used to share data or information related to IPCP efforts. The types of meetings mentioned by hospital site teams are listed in Table 5.

Table 5. Examples of meetings used to engage physicians in IPCP

Types of Meetings

Nursing staff meetings

Nursing leadership meetings

ED directors' meetings

ED medical meetings

Medical \& nursing leadership meetings

Emergency services meetings

Nursing \& physician staff meetings

Hospitalists meetings

Hospital-wide medical staff meetings

ED physicians medical staff meetings

\section{How they engage physicians in IPCP}

Medical Director \& Physicians invited to join

All Physicians invited to join

ED nursing \& medical director meet one-on-one at least weekly

ED director \& ED nurse manager (if agenda involves nursing) attend

Physicians meet with nursing \& administrative leaders

Physicians from different departments attend

Used to share data \& ED related issues

ED director comes to share data \& ED related issues

Medical director shares ED data will all hospital physicians

Hospitalists attend 
Meetings that were described as most successful involved medical and/or nursing leadership. These meetings have resulted in positive working relationships between physicians and nursing leadership. One site noted that these meetings allow nurse leaders to communicate with physicians about issues brought to managers and coordinators by nurses. The most successful meetings appeared to be those between ED medical and nursing directors. Several sites described these regular meetings as crucial to IPCP efforts. Based on experience with many different hospitals, the Director of Clinical Services for the physician contract company at one hospital stated that regular, weekly or daily meetings between these directors is the most important step toward better IPCP in EDs.

Frontline physicians were less likely than leadership to attend regular meetings, which hindered their engagement in IPCP. Physicians only sporadically participated in nursing staff meetings at one site, and it was only the medical directors that attended. At a different site, a monthly meeting for physicians and nurse leaders, called "Coffee with Coordinators," began with sparse participation and is now no longer attended by physicians. The site team noted that the initial meetings were positive and productive and focused on improving the working relationships between nursing staff and providers. They also noted that these meetings contributed to a closer relationship between physicians and leadership, which spread to the rest of the unit. Despite the value of holding frontline physicians in meetings, regular meetings appear to be difficult to sustain.

Using physicians' existing meetings as an opportunity to share data and information may be more successful than asking physicians to attend additional meetings. One nurse leader or other representative can attend regular physician meetings for this purpose. At one site, information related to ED throughput data and quality scores is shared in many different types of 
meetings. For example, ED information is shared in regular hospitalist meetings. The variety of meetings used to communicate ED data, such as patient hold hours, has helped with intraphysician collaboration throughout the hospital as well as IPCP.

Huddles. Three of the ED site teams discussed physician engagement in daily huddles. Two of these teams described huddles involving frontline staff and clear physician participation while another site admitted that they have not included physicians. Huddles occurred on the ED units at different times during the day and week. One site described daily morning huddles while another reported that huddles occurred in the morning, afternoon, and evening.

Successful huddles included information sharing or other elements relevant to physicians. Morning huddles at one site were used to give daily updates on available services, review staffing, and introduce the resident working that day. This site has also added a process improvement element so that staff and physicians can bring up issues that may be easily addressed or fixed. Team members from another site stated that they have morning, afternoon, and evening huddles that include physicians. The site team indicated that they allow ED physicians to use the huddle as an opportunity, called "Medical Minutes," to share information with the rest of the staff. For example, some physicians discuss a new evidence-based practice while others may share their preferences related to patient care during the upcoming shift. This site also indicated that they take advantage of their workspace to include physicians. Huddles are held at the ED nursing station where the physicians are working.

Huddles are not as effective at engaging physicians in IPCP if they are not held regularly or if physicians are not included. One of the sites noted that regular huddles primarily occur during weekday mornings and they have struggled to maintain this practice at night and on weekends. To fill this gap, they now have the charge nurse run the huddles rather than an 
administrative coordinator that only works on weekdays. Team members at a third site described using their regular nursing change of shifts as huddles. These huddles have succeeded in including others, such as security staff. The site team described provider participation and the use of "Medical Minutes" but admitted that advanced practice providers, not physicians, typically participated.

Education. Three of the ED site teams described opportunities for physician engagement in interprofessional education. Most of the examples were provided by one site and included hands-on or gaming simulations as well as classroom courses. Some educational opportunities were provided specifically for the ED unit while others were offered for the entire hospital, medical residents, or the Shaping Systems project sites.

The educational activities that were most successful involved simulation. One hospital reported that they use a mobile simulation lab to provide onsite simulations for all staff. They block off an ED bed and physicians can participate along with other staff during their normal work hours. As part of the Shaping Systems collaborative, three sites used the simulation game Friday Night in the ER ${ }^{\circledR}$ as an interprofessional educational experience. This simulation game encourages IPCP and a system approach to patient care. All of these sites reported some physician participation in this gaming simulation event.

Educational events that were not as successful in engaging physicians in IPCP were classroom based. One site team described hospital-wide classes, such as a language of caring class and TeamSTEPPS, that are mandatory for physicians as well as other staff. While these courses help promote IPCP by teaching the same topics to all professions, the site team admitted that the physicians attend classes with only other physicians. These classes do not allow different professions to learn these concepts together. This site also encouraged nursing staff to 
attend regular on-site medical resident classes. These classes are taught by physicians and cover a variety of topics such as evidence-based antibiotic use. These courses are designed for physician participation; however, nurses and other professionals do not attend due to scheduling conflicts and patient care needs.

Policy or process. Team members from all four sites shared at least one example of a policy or process that assisted with physician engagement in IPCP. The use of policies or processes to direct IPCP between physicians and other professionals involved documentation, roles and responsibilities, communication, and staffing. The nature of the IPCP that resulted from these policies or processes varied but all the examples given were described as successful.

One ED director explained that their boarder patient policy had been changed to better explain the collaboration that needs to occur between nurses and physicians. He stated that the new policy clarifies documentation and responsibilities for both professions. Another site has added a sepsis protocol to dictate the roles and responsibilities of physicians and nurses while caring for patients at risk for sepsis. This site has also developed a rapid admit process that directs the responsibilities and collaboration that needs to occur between different types of providers, such as ED physicians, intensivists, and hospitalists, to get patients admitted and transferred out of the ED quickly. Team members from a third site stated that they use a standardized communication tool (Situation-Background-Assessment-Recommendation) that works well for communication and collaboration with physicians. The fourth site team discussed a change in their ED staffing process. After nurses changed their staffing patterns to better match patient volume needs, physicians agreed to follow the same changes so that their staffing patterns would be aligned. 


\section{Logistical - Making it as easy as possible for physicians to participate. All four ED}

site teams reported strategies related to the logistics of involving physicians in their IPCP activities. These strategies focused on distance technology, scheduling/timing, location, and managing and communicating requests for physician participation. The site teams used these strategies to address practical matters to encourage engagement and make it as easy as possible for physicians to participate in their IPCP events.

Distance technology. Members of two site teams discussed their use of distance technology to allow physicians to take part in IPCP activities when they were not at the hospitals. Both sites noted that the use of video or phone conferencing has improved physician participation in meetings or other events. At one site, three physicians attended an educational inservice being presented to nurses and other physicians at the hospital by using video conferencing from home. The medical director at this site was also able to join a learning session at a Shaping Systems learning collaborative by using video conferencing. The second site spoke generally about the use of conference calls and "other technology" as a method for successfully getting physicians to attend meetings.

Scheduling/timing. Two of the ED site teams mentioned strategies they use for scheduling IPCP events to encourage more physician participation. These strategies involved targeting the best times of the day and giving physicians adequate advance notice. Using these strategies appeared to work well for scheduling onsite educational events. One site described scheduling onsite interprofessional education activities, such as simulations, at times when their unit was typically not busy. They also made sure to advertise the activities ahead of time to all staff and physicians. Team members reported that these strategies have resulted in better physician attendance. A second site mentioned using the same strategies for meetings. Their ED 
director stated that he looks to schedule meetings at the best time of day for physicians. He also notifies physicians of when meetings are held to encourage attendance. This site team acknowledged mixed success with these strategies stating that they still struggle to get physicians to join meetings. An ED nurse at one site stated that 'it's not that [physicians] don't want to help, it's more time management, so how can we help that will work for them in their time frame or schedule."

Location. Three of the site teams discussed ways in which the choice of location affects physician participation in IPCP activities. Scheduling events or meetings at the hospital was a universally reported strategy that improves physician attendance. Holding events in the ED is even more effective for getting physician participation. One site team reported that they hold meetings on the unit so that staff and physicians who are working can attend. Site team members admitted that physicians only attend if they are staffing during that time. Another site team discussed several examples of physician participation that resulted from events occurring onsite. They mentioned the interprofessional education simulations that occur on the unit which result in physician participation. The team also mentioned two Shaping Systems' events that took place at their hospital, a quarterly learning collaborative and a Friday Night in the $E R{ }^{\circledR}$ simulation game. One to two physicians attended each of the Shaping Systems events and the site team believed that this would not have occurred if the activities had been held elsewhere. The third site team also mentioned an onsite Friday Night in the $E R{ }^{\circledR}$ event at their hospital that led to participation by a physician. While scheduling activities onsite does not guarantee physician engagement and participation, it appears to increase the likelihood that some physicians will attend. Physician participation is lower for events that occur further from the hospital, such as the Shaping Systems learning collaboratives or other conferences that require more travel time. 
Managing and communicating requests. All four of the ED site teams discussed ways in which they request participation from physicians. These strategies involve prioritizing needs for physician participation, respecting physicians' time, and communicating requests for physician participation. All of these strategies were described as improving physician engagement in IPCP activities. A summary of strategies is provided in Table 6.

Table 6. Summary of successful strategies for managing and communicating requests

\begin{tabular}{ll} 
Strategy & $\begin{array}{l}\text { Details/Descriptions/Considerations } \\
\text { Is a physician needed for this project? Does } \\
\text { physician practice affect this issue? }\end{array}$ \\
Prioritize requests & $\begin{array}{l}\text { Preparation for and consolidation of } \\
\text { physician-related issues }\end{array}$ \\
Respect physicians' time & $\begin{array}{l}\text { Tells physicians exactly what is needed from } \\
\text { them and increases accountability }\end{array}$ \\
Assign specific tasks & $\begin{array}{l}\text { Communicates that they are needed } \\
\text { Be persistent }\end{array}$ \\
Explain "why" & $\begin{array}{l}\text { Tell them why you need them to attend a } \\
\text { meeting or participate in a project } \\
\text { Communicate need for physician participation } \\
\text { to several or all physicians }\end{array}$ \\
\hline
\end{tabular}

Members from three teams brought up the need to respect and prioritize requests for physicians' time. One site team stated that they pay attention to which issues require physician participation and prioritize their involvement. They avoid involving physicians in projects such as bed management or improving patient handoffs to nursing homes because these issues are not affected by physicians' practice. Similarly, another site team recognized that physicians were not always needed at events such as daily leadership bed huddles.

Site teams also discussed ways in which physicians' limited time can be respected. One team reported that they prepare for meetings ahead of time to identify and outline all the issues 
they need to discuss specifically with physicians. This preparation results in more productive meetings and allow them to successfully address issues with physicians. Similarly, two other site teams discussed organizing all physician-related information and presenting it during one consolidated block of time. This strategy was suggested for the two-day Shaping Systems learning collaboratives to encourage participation for at least one or part of one day. This strategy would have benefited the physicians from these sites who were only able to attend one day of a Shaping System learning collaborative.

The fourth site mentioned two additional strategies that have helped them to increase physician engagement in their IPCP efforts. The site team suggested assigning specific tasks to physicians in order to increase accountability. The other strategy is to "be persistent" when requesting physician participation. According to one team member, this strategy works because it "conveys the message that 'you are needed' to the physicians." Similarly, a team member from another site stated that it is important to explain to a physician "why they are needed at a meeting or for a project."

Two of the site teams discussed communication with physicians. One ED director mentioned that he sends the same ED unit email communications to all staff and physicians. He anticipated complaints from physicians related to the large number of emails but reported that this has not occurred. Another site's ED director noted that she has four key physicians to whom she sends all communications and requests. She states that by communicating needs and issues to several physicians she is more likely to get at least one physician involved. 


\section{Interpersonal - Who and how you work with others to promote and facilitate}

physician participation. Each of the four site teams discussed strategies related to who and how they work with to get physician engagement in IPCP efforts. Specific ideas for using relationships to improve physician participation were related to building personal relationships, using Appreciative Inquiry, working with leadership, and working with others who are not directly part of their ED site teams. These strategies were utilized to leverage personal relationships to promote and facilitate physician involvement in IPCP.

Building personal relationships. Three of the site teams discussed effective strategies they and their staff have used to enhance their relationships with physicians. These approaches for relating to physicians are summarized in Table 7.

Table 7. Approaches to building personal relationships with physicians

\section{Use first names}

Address concerns directly with physicians

Personal invitations to participate

Social events outside of work

Inquire about physicians' interests

Consider physicians' perspective

One site team reported that their nurses are making efforts to call physicians by their first name. Staff are also being encouraged to address physicians directly about concerns or deviations from standard practice rather than using administrative channels. Two of the site teams noted that they have seen improved participation when physicians are personally invited and reminded to attend or be involved. One site member noted that getting physicians to participate in their daily huddles is "not a hard thing to do - you just have to invite them." 
Two of the site teams also mentioned the benefits of spending time together outside of work. One team noted improved dialogue and personal relationships between their ED and hospitalist medical directors after they spent time together at a conference. Similarly, another site team said that the relationships between their physicians and nurses benefit from participating in social events outside of work. A third site has begun to schedule staff and provider social events outside of work.

Three of the site teams reported that they have tried to discover and match physicians' interests with patient care issues that need to be addressed. Taking the time to learn more about physician interests has allowed these teams to find the right physician champions to become involved and communicate their efforts to other physicians. Two of these site teams stated that their physicians are more likely to participate in efforts when they are interested in the issue or they can see a benefit to their practice if the issue is addressed. The Director of Clinical Services for the physician contract company at one site confirmed that taking the time to discover physicians' interests is a helpful approach to finding the right physician champions. According to the ED director at a larger hospital who was a guest speaker at a learning collaborative, understanding physician engagement from the physician's perspective is very important. He stated that site teams should "put their hat on" and "talk directly to them about it and don't beat around the bush."

Using Appreciative Inquiry. Appreciative Inquiry is a strategy that was introduced to site teams through the Shaping Systems collaborative. This change management strategy focuses on what is working well and then doing more of it. Two of the ED site teams specifically discussed using this tool with physicians. One of the site team members stated that when they used Appreciative Inquiry during a meeting with physicians, their positive approach 
to addressing sensitive issues was well received. A nurse coordinator from another site noted that she has tried to be more positive when addressing physicians. For example, she has been telling physicians when they are doing a good job and meeting unit goals at the end of each shift.

Working with leadership. All the ED site teams addressed the importance of leadership support to improve IPCP with physicians. Two site teams discussed ways in which relationships with leadership have facilitated physician engagement. Working with leaders is a successful strategy when leadership is supportive of ED teams' IPCP efforts and leadership turnover is low.

Site teams described ways in which they have gained leadership support. Members from one of the sites spoke generally about getting administrative support for the IPCP work they have done. They stated that meeting with administration has enabled them "to get physicians on board with improving and meeting performance metrics such as ED patient throughput.” Another site team has also shared ED data with hospital leadership to show the need for more IPCP efforts to improve care. A third ED site team has communicated the work they have done in the Shaping Systems collaborative with their leadership in order gain more buy-in and support for physician engagement in IPCP.

Other teams cited specific examples where leadership facilitated physician engagement. One site reported that their medical director has participated in process improvement meetings in which they share goals for patient care issues such as length of stay. The medical director has then met with providers to discuss their impact on goals as well as sent out information to providers about the status of improvement efforts. One chief nursing officer was involved in getting physicians to participate in an onsite Shaping Systems event.

The ED director at one site described the hiring of a hospital medical director as key to his ability to engage physicians in IPCP efforts in his ED. He noted that previous efforts to use 
leadership support as a strategy for engaging physicians had been unsuccessful. Other sites also mentioned that turnover in key leadership positions has led to ineffective use of leadership to support physician engagement in their EDs.

Working with others. Two of the site teams described ways in which people from outside their team have helped to promote and facilitate IPCP in their EDs. Examples of other professionals used to support physician engagement in IPCP efforts are listed in Table 8.

\title{
Table 8. Examples of other professionals used to improve physician engagement
}

\author{
Physicians from outside the hospital \\ ED professionals from other hospitals \\ Psychiatry director \& educator \\ Social workers \\ Liaison from physician contract agency \\ Chief Process Improvement Officer
}

Both site teams discussed using Shaping Systems team members and participants to encourage physician engagement in IPCP activities. Both site teams noted that the physician lead for the Shaping Systems project had reached out to encourage physician participation in the Shaping System activities or learning collaboratives. As a result, both teams had physicians attend learning collaboratives and one of the sites had physician participation in two different onsite Shaping Systems activities. One of the site teams noted that it was "good for physicians to hear ideas from teams at other hospitals" and that it encouraged them to want to work on issues in their own EDs.

Team members from one of these sites also shared multiple examples of working with different professionals outside the ED to support physician engagement in their IPCP efforts. They included the director and educator of psychiatry as well as social workers in their efforts to 
improve their discharge process for psychiatric patients. These professionals provided the education and support needed to get the ED physicians more involved. Additional support for physician engagement has come from the Director of Clinical Services for the contract agency that provides ED physicians for the hospital. She has served as a liaison and source of information for the ED team and is interested in helping the hospital improve their patient care as it affects her company's goals. This ED team has also used an engineer with a background in process improvement to help with their improvement efforts. As a non-clinical staff member, he provides a neutral point of view for physicians and other clinical staff and has gained their respect after working with them for over a year to improve their patient care processes.

\section{Quality improvement related - Working with physicians on patient care}

improvement efforts. All four site teams spoke about strategies for engaging physicians in their quality or process improvement efforts. Strategies for getting physicians engaged in these overall efforts included getting participation in specific projects or initiatives, sharing data and goals, sharing success, and including goals in physician contracts. The ED teams all reported participating in many improvement projects and initiatives at their hospitals. They also spoke of the need to include physicians to make meaningful improvement in patient care.

Participation in specific projects or initiatives. Two of the site teams shared examples

of physicians being involved in individual improvement projects. One site held a rapid improvement event to work on patient throughput in the ED. The ED director stated that the ED medical director and two physicians joined the four-day event and felt that it was very productive. This strategy allowed the ED team to concentrate their work with physicians into several days rather than meet over a longer period. Another site stated that they are typically able to get physician representation in their Lean improvement projects based on the physicians' 
interests. The team noted that they also include physicians based on relevance to the project. For example, a hospitalist was included in an improvement project related to admissions. One of their strategies for engagement is to include the medical director at the beginning of the project and then focus on which roles need to be included in the work as the project continues. According to one team member, the medical director is usually able to speak from the physicians' perspective. Involving physician leadership early in the process, limiting the time needed for physicians to participate, and focusing only on the physicians needed and interested in specific issues contributed to the successful use of this strategy for these site teams.

Share data and goals. All four of the ED teams reported that they share patient care data and goals with their physicians. One site noted that they provide physician-specific feedback on performance for department metrics that impact larger hospital goals. The nursing director works with the medical director to focus on how they can help low performers improve. Similarly, another site team stated that they show productivity reports to physicians and the Chief Medical Officer and then meet with outliers. One team member described working with a provider that was not meeting her goals to help her succeed and to share the nurses' perspectives on how she can improve. This site also reported sharing other data with all staff and physicians, like patient volumes, in order to get buy-in for changes in staffing patterns. A third site team stated that "we share data with providers to get buy-in and to get them to see their part and what they control." The team was unclear as to whether this information was shared with all physicians or just the medical leadership. The fourth team discussed communicating hold hours with the hospitalist group as well as their staff. They reported that sharing this information in different meetings with all groups has contributed to improved physician participation in efforts to improve ED patient throughput. According to one team member, "people respond to what you 
measure." Successful use of this strategy includes support for low performing providers and sharing data to other staff and physicians as needed. Use of this strategy improves buy-in from providers for participation in patient care improvement efforts.

Share success. Two of the ED site teams emphasized the role of sharing success in improving physician engagement. One site team noted that they let physicians know about the progress they are making to improve problems in the ED. They noticed increased physician engagement as the physicians have seen the successful results of improvement efforts. Another site team shared a number of their accomplishments with one of their physicians during a Shaping Systems learning collaborative. This physician expressed the inspiration she had from seeing their progress and has become more interested and engaged in both the Shaping Systems collaborative and her ED's improvement efforts.

Include goals in physician contracts. According to three of the site teams, performance goals are part of their physicians' contracts. One site team reported that goals for physicians have been written into their contracts and that their metrics are reported weekly. As a result, one team member stated that the physicians now "feel the heat" and "they own it." An administrative executive at this site noted that hospital goals and expectations regarding participation in meetings and committees should all be included in physician contracts. This ED team listed "incentive based providers" as one of their goals on their Shaping Systems vision board. Another team reported that performance measures were included in their physicians' contracts. They have noticed more physician engagement in improving patient care issues as physician compensation is now based on their quality metrics. A third team announced that quality and process metrics had recently been linked to physician bonuses like they have been to nursing bonuses. The team members said they were hopeful that this would improve physician 
engagement in their IPCP work. While these teams were not responsible for incorporating goals into their physicians' contracts or reimbursement, they have been able to learn more about what physicians are accountable for and to leverage these arrangements to encourage participation in improvement efforts. 


\section{CHAPTER 6: DISCUSSION}

The purpose of this project was to identify barriers, facilitators, needed resources, and successful strategies to improve physician engagement in interprofessional collaborative practice in four rural EDs participating in a project designed to build IPCP capacity. Seven major themes were found to describe the contextual factors (barriers, facilitators, needed resources): employment arrangements, scheduling, competing priorities, leadership, work environment, resources, and knowledge. The strategies that were identified were organized into four general categories: structural, logistical, interpersonal, and quality improvement related.

Figure 3 presents a framework for how this study's findings may inform efforts to improve physician engagement in IPCP and thereby improve relational coordination (RC) and care outcomes. The framework explains how the strategies found in this project impact RC as well as contextual factors that influenced both the execution of strategies and their impact on RC. 


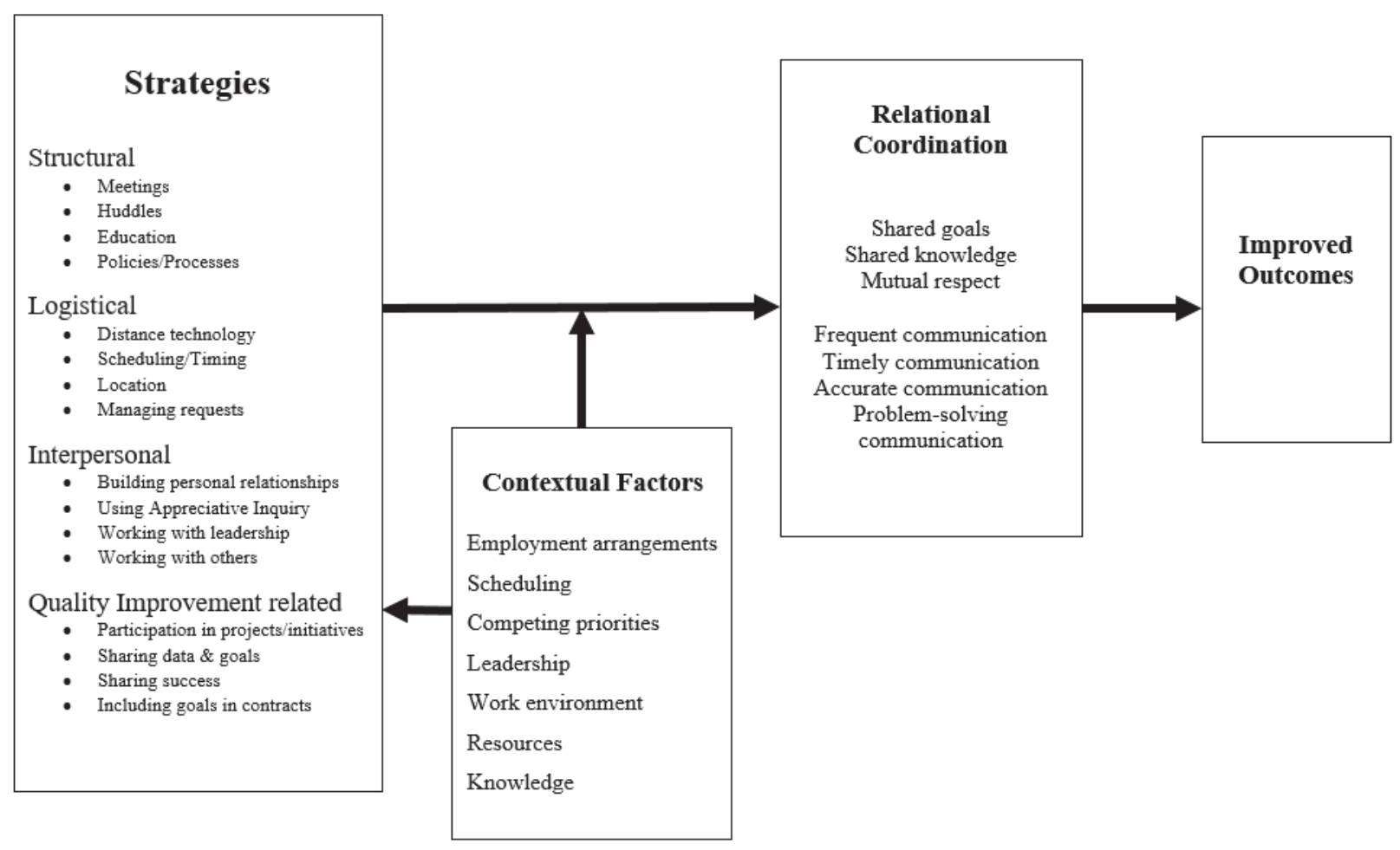

Figure 3. Framework for improving physician engagement in IPCP

Contextual factors. Many of the contextual factors identified in this project were similar to those reported in prior literature on physician engagement in IPCP and organizational initiatives. Employment arrangements have previously been found to be barriers to physician participation in collaborative efforts (Herbert et al., 2007; Poochikian-Sarkissian et al., 2008) and quality improvement initiatives (Taitz et al., 2012). Scheduling difficulties specifically related to conflicting patient care responsibilities were also noted in a report on physician engagement in health care development (Lindgren et al., 2013). Aspects of the work environment also have been identified in prior studies. The use of joint workspaces, for example, has been found to affect nurse/physician communication (Manojlovich, 2010) and IPCP in a primary care setting (Morgan et al., 2015). Other aspects of work environment frequently discussed in the physician engagement literature include professional factors related to physicians' desire for autonomy and culture of independence over teamwork (Byrnes, 2015; Lindgren et al., 2013; Rosenstein, 2015; 
Taitz et al., 2012). This project's findings suggest that barriers related to employment arrangements, such as lack of compensated time, scheduling, and competing priorities, have a greater impact on physician engagement than the work environment. This study contributed a new finding related to the role of nursing leaders' and other staffs' lack of knowledge of physician arrangements as a barrier to physician engagement.

Strategies. Many of the strategies identified in this study also have been identified previously. Structural strategies, such as meetings (Minamizono et al., 2013; Suelflow, 2016), interprofessional ward rounds, interprofessional or tailored education programs (Rosenstein, 2015; Suelflow, 2016; Tang et al., 2013), formal policies or procedures (Poochikian-Sarkissian et al., 2008), communication protocols, and scheduling that supports workgroup continuity (Kaissi, 2014; Lindgren et al., 2013) have all been reported as strategies for physician engagement in IPCP. The interpersonal strategy - leadership and management support - identified in this project has been described as an approach to improving physician engagement (Fitzgerald \& Teal, 2003; Liebhaber et al., 2009). Other interpersonal strategies mentioned in the literature include open communication and taking physicians' needs and concerns into account (Rosenstein, 2015; Skillman et al., 2017; Spaulding et al., 2014; Stark, 2014; Swenson et al., 2016). Many of the quality improvement related strategies recognized in these findings have been identified in the literature on physician engagement in improvement projects or initiatives. Previously reported strategies include the use of data (Kaissi, 2014; Liebhaber et al., 2009; Sears, 2011) and feedback on quality metrics (Suelflow, 2016) as well as using common purpose and shared goals (Kaissi, 2014; Kreindler et al., 2012). The specific use of formal contracts and mutual accountability for outcomes was also noted by ED site teams and in a literature review on 
administrative strategies for improving provider engagement in hospital quality initiatives (Suelflow, 2016).

While many of the structural and quality improvement related strategies identified in this project were noted in the physician engagement literature, logistical strategies and many interpersonal strategies were not found. Project site teams identified several strategies designed to make it easier for physicians to participate, such as using distance technology. The logistical strategies identified in this project address many previously identified barriers to physician

engagement. Socializing outside of work, personal invitations to participate, using Appreciative Inquiry, and working with others were all interpersonal strategies not previously addressed in the physician engagement literature.

\section{Limitations and Future Work}

Limitations to this project include the use of a convenience sample. Working within a larger project, the DNP student investigator was limited to the ED site team members that were able to participate in the Shaping Systems events used for data collection for this project. The participating hospitals decided who and how many participants were on each team. Teams had different numbers of members and were composed of different roles. This limitation affected data gathered by the interviews more than other events. The team members that participated in each interview changed between year one and year two for each of the teams. In the case of one site, the team coordinator was not available for the second-year interview. The person interviewed noted that she was not as knowledgeable as the coordinator about some of the questions asked.

The use of convenience data collection was also a limitation. As mentioned, the DNP student investigator primarily used existing Shaping Systems events to obtain information on 
physician engagement. Data on physicians and physician engagement was only gathered when it came up as part of site teams' general discussion at these events. Data collection that was focused solely on physician engagement was only done during the second-year interviews and during a site visit to one ED to shadow a physician. Using Shaping Systems events for data collection also led to unequal access or exposure to the four site teams. During this project, the Shaping Systems team made two site visits to one hospital and held a learning collaborative at that same hospital. The DNP student investigator was therefore able to visit this hospital three times. Another site was visited once and two of the sites received no site visits during the time of this project. However, all four sites were represented at the learning collaborative sessions and interviewed twice.

Another factor that may have affected data collection on physician engagement was the presence of physicians during data collection. Physicians attended some of the learning collaborative sessions and were present during parts of the site visits. During the last learning collaborative, a nurse from one site team spoke to the DNP student investigator privately about her inability to speak up during a discussion about physician engagement because one of the ED physicians from her hospital was in attendance. While many participants spoke during times when physicians were present, others may have withheld information they did not feel comfortable sharing in front of physicians with whom they worked.

Lack of physician input was another limitation of this project. While some physicians were involved in data collection, the majority of information about physician engagement came from nurses. Building nursing's capacity to lead IPCP was one of the objectives of the Shaping Systems project; therefore, their perspective was valuable for this project. However, the need to consider physicians' perspectives, concerns, and agenda have been noted both during this project 
and in the literature (Skillman et al., 2017; Stark, 2014; Suelflow, 2016). Other stakeholders that were not included in this project are advanced practice providers (APPs), such as nurse practitioners and physician assistants. All four of the participating sites use APPs who work closely with physicians in their EDs. The scope of this project limited data collection to physicians; however, APPs should also be included in IPCP efforts in EDs. In addition, these providers could play an important role in coordinating with physicians, nurses, and others to gain buy-in and support for IPCP efforts.

In the future, ED site teams should continue to recognize barriers to physician engagement that are specific to their settings. They should adapt and develop strategies based on identified barriers as well as those reported in this project. Strategies should continue to be evaluated and results reported so that time and effort can be placed on those that are most successful. Site teams should also work with physicians to learn more about their perspectives on physician engagement as well as their concerns and interests for improving patient care. Finally, ED sites should consider including other team members that work closely with physicians, such as APPs and scribes, in their efforts to improve physician engagement.

\section{Project's Impact on Participating ED Site Teams}

Participating site teams benefitted from this project's specific focus on physician engagement. By the end of this project, each of the participating ED site teams had described insights or experiences that improved physician engagement in IPCP efforts at their hospitals. The need to learn more about their physicians' employment arrangements was a key discovery for some ED teams. During an interview, the ED director from one site was unable to answer questions related to physician responsibilities, compensation, time allotment, and accountability. During this exchange, he said "just in hearing me talk, I should be reaching out to [the medical 
director] and saying hey, how is this structured?" The insight gained from this discussion led him to learn more about his hospital's ED physician employment arrangements in order to guide future strategies for gaining physician engagement when needed.

Focusing on physician engagement motivated one site team to reach out beyond their medical director to another physician in their ED who expressed interest in the Shaping Systems project. After attending a learning collaborative and seeing all the progress the site team had made on different issues and projects, this physician became a champion for this teams' efforts and has remained engaged in their local improvement activities. This ED team found that it is important to reach out to multiple physicians to find one who will engage. Assessment and feedback related to this project encouraged site teams to examine the level of physician participation in their IPCP efforts. One team acknowledged that physicians were no longer attending huddles, while another realized that physician attendance to meetings with nurse leaders had dropped. These site teams recognized the need to renew their efforts to get physicians involved. Overall, the inquiry and discussion related to this DNP project prompted the participating ED site teams to take steps toward improving physician engagement in IPCP efforts in their hospitals.

\section{Implications for practice}

Engaging physicians is becoming even more important given the changes to health care delivery systems that focus more on quality of care and realign reimbursement with outcomes (Skillman et al., 2017; Suelflow, 2016). The strategies identified in this project can be used by

nursing leaders or anyone interested in engaging physicians in IPCP to improve patient care in a rural ED setting. This includes quality or process improvement projects or the implementation of new evidence-based practices for routine patient care. In addition, the contextual factors 
described in this project can inform others who may be faced with the same or similar issues in their settings. Different strategies may be developed to remove barriers or leverage existing resources or facilitators. The removal of barriers has been identified as a strategy for engaging physicians in hospital quality initiatives (Suelflow, 2016). Based on the findings from this project, a toolkit has been created for others who are interested in improving physician engagement in IPCP in their EDs. The toolkit includes strategies as well as the contextual factors that influence their applicability and success and is included in Appendix B.

\section{Dissemination}

As noted in the results section, the findings from this project were shared with participating site teams. This served two purposes: to confirm the findings, but also to share them with all teams so that they could learn from what other sites are doing to improve physician engagement in their IPCP efforts. After this information was shared with the sites at a regular learning collaborative session, the presentation was posted on the collaborative's website for future reference. This website was designed as a continuing resource for the hospitals participating in the Shaping Systems learning collaborative. The toolkit will also be added to this website.

Sharing the results with the key stakeholders in this project was the primary dissemination objective; however, other mechanisms for dissemination will be pursued. The findings from this project will also be disseminated in a poster presentation at a nursing research and quality conference sponsored by UNC Health Care. The target audience for this conference is nurses and others interested in learning more about improving patient outcomes using strategic quality projects and research. The DNP student investigator for this project will also work with committee members, including the principal investigator of the Shaping Systems project, to 
publish these findings. Publication may be in conjunction with other findings from the larger Shaping Systems project once it has been completed.

\section{Conclusion}

Interprofessional collaborative practice can positively impact the quality of patient care in EDs by improving communication and collaboration among members of the healthcare team. Physicians are integral members of this team and need to be engaged in IPCP activities and efforts; however, physician engagement in IPCP is challenging. This project identified several strategies that have been used in rural EDs to get physicians to participate in IPCP efforts. Contextual factors that influence physician engagement were also identified. These contextual factors and strategies can be used to inform efforts to improve physician engagement. While additional physician input would strengthen these efforts, the strategies outlined in this project create a foundation for improving physician engagement in IPCP in rural EDs. 


\section{APPENDIX A: TELEPHONE INTERVIEW GUIDE}

Date:

Hospital:

Profession:

Position in the hospital:

Thank you for taking the time to talk with me. I have some additional questions specifically related to physician participation in the project and in any interprofessional collaborative efforts you have implemented at your hospital.

1. You have discussed several projects and strategies developed or implemented as part of your facilities' involvement in the Shaping Systems project. Can you describe physician participation in these efforts? How often have physicians participated in these efforts? Have physicians from your hospital participated in any Shaping Systems activities? If so, what was their level of participation or how often have they participated?

2. What are some of the factors that have helped make possible or encouraged physician participation in either Shaping Systems activities or the interprofessional efforts at your hospital?

3. Can you describe challenges in your setting that discourage or impact the level of physician participation in the Shaping Systems project or interprofessional efforts at your hospital? 
4. Are there any resources in your setting that you feel have been necessary to get physician participation? Are there any resources you believe would increase the level of physician participation if they were available in your setting?

5. What strategies has your hospital used to get more physician participation in the Shaping Systems project or other interprofessional efforts at your hospital? Have these strategies been successful? 


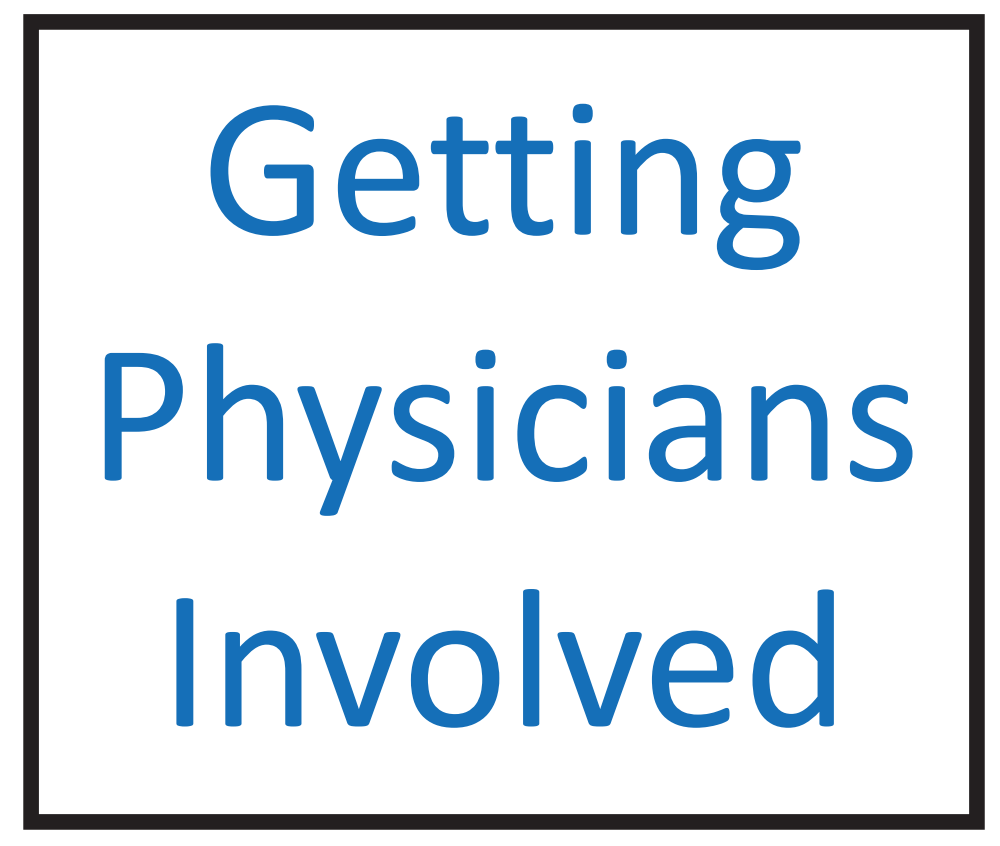

Ideas for improving physician engagement in interprofessional collaborative practice efforts in your rural ED to provide high quality patient care

- Include physicians in structures already in place at your hospital

- Make it as easy as possible for physicians to participate

- Think about who and how you work with others to promote \& facilitate physician participation

- Work with physicians on patient care improvement efforts 


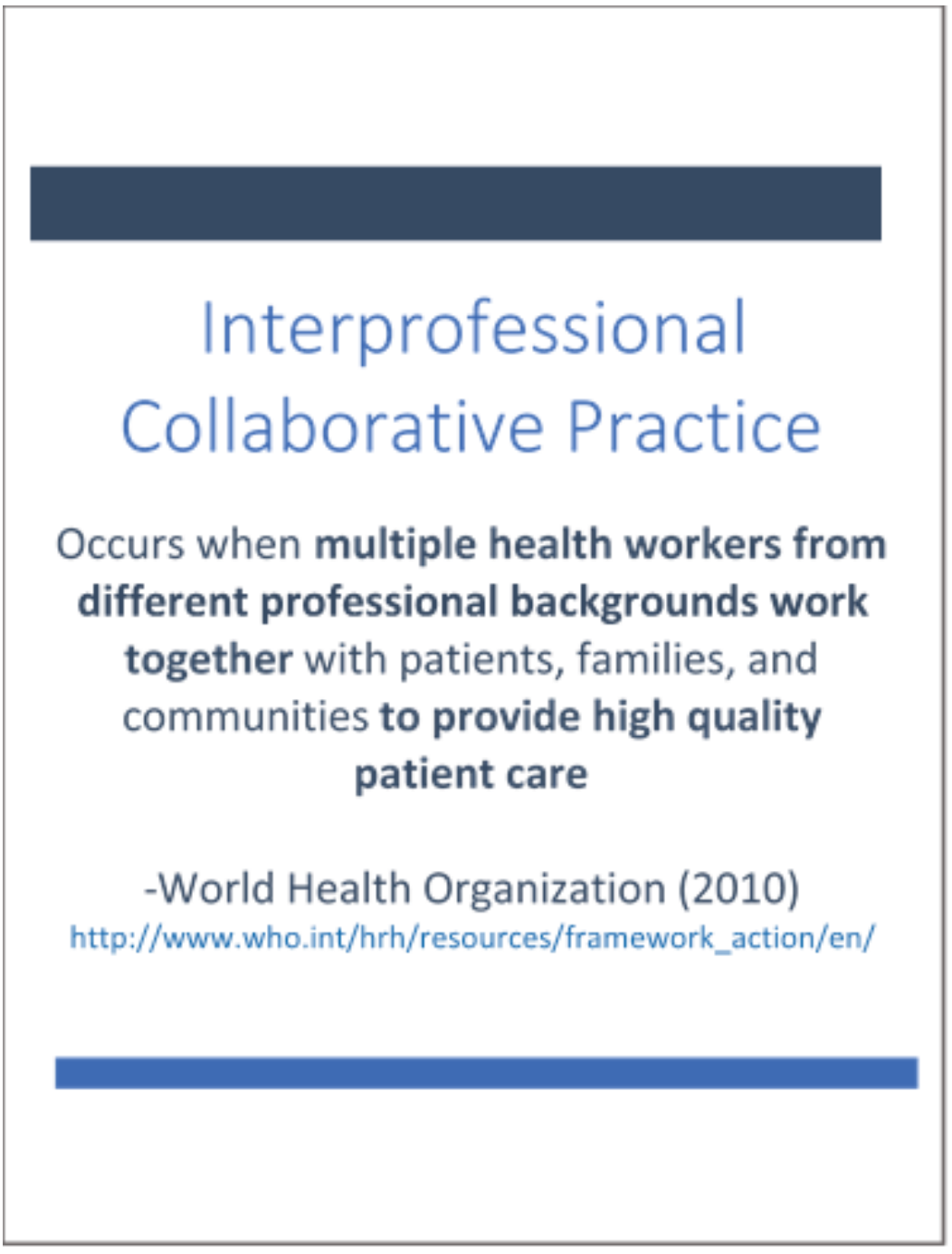




\section{Use these strategies to hardwire physician engagement}

\section{Meetings}

- Involve leaders in meetings rather than front-line physicians \& staff because they typically have more time and better participation

- Use front-line physician \& staff meetings to share ED information

- Share important ED quality \& process data

- Bring staff concerns \& issues to physicians

- Share ED information with all hospital units \& physician specialties who impact the ED

The most important meetings for ED physician engagement are regular, frequent meetings between $E D$ medical and nursing directors

Challenges to address: Scheduling, Location, Limited time allotted to physicians for administrative activities

Tips: Be prepared \& address all physician issues in one block of time. Consider onetime or limited series of meetings to deal with acute physician-related issues. 


\section{Huddles}

- Hold regularly- every day, preferably several times per day

- Include as many team members and different professions as possible

- Build into the routine workday/ shifts to allow for better participation from front-line staff and physicians

- Choose someone in a role that works every day and shift of the week to lead

\section{Use as an opportunity to address small improvement} suggestions or updates on improvement projects.

Challenge to address: Should not be the regular nursing change of shift report

Tips: Allow physicians to share information with staff - "Medical Minutes." Ideas include practice updates or physician preferences for the upcoming shift.

\section{Education}

- Teach teamwork, for example

- Teach TeamSTEPPS classes for physicians \& staff together

- Use simulations to promote interprofessional collaborative practice, for example

- Conduct hands-on simulation activities on the unit during a regular workday

- Increase system-awareness, for example

- Offer Friday Night in the $E R^{\circledast}$ simulation game

Best when professionals are learning concepts together, not just learning the same material separately

Challenges to address: Aligning schedules, pulling staff \& physicians from patient care 
Tips: Take advantage of hospital-wide mandatory classes and medical resident classes

\section{Policies/Processes}

- Use these to direct documentation, roles \& responsibilities, communication and staffing

- Policies or processes can indicate who should document or do certain tasks, for example

- Policy that dictates documentation by different professionals for patient boarding in the ED

- Protocol that outlines roles \& responsibilities of physicians \& other staff for care of patients at risk for sepsis

- Policies or processes that require communication \& collaboration among physicians and other staff, for example

- Situation-Background-Assessment-Recommendation (SBAR)-standard communication that directs how communication should occur between different professionals

- Staffing process that aligns physician and nursing schedules

Best policies \& processes are those that require communication \& collaboration among physicians and staff

Challenges to address: Physician \& staff buy-in, culture, resistance to change, electronic health record systems that do not allow for communication between roles

Tip: Look for examples from other EDs or settings 


\section{Address practical matters to encourage engagement}

\section{Distance Technology}

- Use video or phone conferencing

- Enables physicians to participate more easily in meetings, quality or process improvement events, educational activities

\section{Helps to counter barriers related to scheduling or location}

Challenges to address: Resources, availability of necessary equipment

Tip: If applicable, take advantage of IT resources available through larger healthcare system

\section{Scheduling/Timing}

- Hold events or activities during, just before, or just after work hours

- Use short time frames - 1-4 day events

- Schedule limited series of meetings

- Advertise ahead of time

- Give plenty of advanced notice

Challenge to address: Pulling staff \& physicians from patient care, including those who work night shift

Tip: Try to avoid times when the ED is busy or typical vacation times of the year 


\section{Location}

- Holding events or activities on the unit is best

- In the hospital or onsite is the next best option

- For larger events, within short driving distance

Challenges to address: Finding space on the unit to hold activities or events, lack of resources, such as ED educator to organize unit events

Tip: Bring in outside resources, such as a mobile simulation lab, if possible

Location can affect scheduling and timing. It is harder for physicians to participate if a large amount of travel time must be factored in.

\section{Managing Requests \& Communication}

- Prioritize requests

- Is a physician needed for this project? Does physician practice affect this issue?

- Respect physicians' time

- Preparation for and consolidation of physician-related issues

- Assign specific tasks

- Tells physicians exactly what is needed from them and increases accountability

- Be persistent

- Communicates to physicians that they are needed

- Explain "why"

- Tells them why you need them to attend a meeting or participate in a project

- Cast a wide net 
- Communicates need for physician participation to several or all physicians

Helps to counter barriers related to competing priorities as well as gain physician buy-in and build mutual respect

Challenges to address: Physicians' lack of knowledge of issues that need to be addressed. Differences in roles \& responsibilities of physicians from other specialties whose participation is needed.

Tips: Gain support from leadership and use them to help make requests and garner engagement. Learn more about physician responsibilities, interests, and concerns. 


\section{Leverage personal relationships to improve physician engagement}

\section{Building Personal Relationships}

- Use first names

- Have staff address concerns directly with physicians

- Make personal invitations to participate in events or activities

- Hold social events outside of work

- Inquire about physicians' interests

- Consider physicians' perspectives

\section{Using Appreciative Inquiry}

- Focus on what is working well

- Address issues with physicians using a positive approach

Creates opportunities to learn more about physician responsibilities or employment requirements so these can be leveraged to gain buy-in \& engagement.

Challenges to address: Culture, resistance to change

Tips: Incorporate interprofessional education into social events outside or at work, such as playing Friday Night in the $E R^{\circledR}$. Recognize physicians for meeting quality or process measures. Personally address physicians at the end of the shift for things like good teamwork or efficient patient care. 


\section{Working with Leadership}

- Gain leadership support

- Share work, data, success with leaders to gain buy-in

- Leverage leadership support to facilitate physician engagement

- Engage physician leaders in improvement efforts and to share information with frontline physicians

Challenges to address: High levels of leadership turnover, competing priorities

Tips: Communicate to leaders why physicians are needed and how efforts relate to hospital or organizational goals

\section{Working with Others}

- Think about others who could help with efforts to improve specific patient care issues

- Psychiatric or behavioral health director or educator, social worker, patient registration, pharmacist

- Engage others to encourage physician involvement

- Let physicians hear from ED professionals and physicians from outside the hospital

- Include neutral roles to help with improvement efforts

- Process improvement engineer, physician contract agency practice consultant

- Use engagement with others to increase buy-in, trust, education

Challenges to address: Aligning schedules, lack of needed resources if hospital does not employ these professionals or have access to others

Tips: If possible, take advantage of professionals either in the larger healthcare system or in outside collaboratives or groups

Relationships with others should be leveraged to improve physician engagement 


\section{Working with physicians on improvement efforts}

\section{Involvement in Specific Projects or Initiatives}

- Take advantage of events with limited time frames when possible, for example

- Rapid improvement events or Kaizen events

- Events can be planned by others and require only 1-4 days of physician engagement

- Take advantage of physicians' interests when identifying a physician champion

- Only involve physicians in projects or initiatives related to issues they impact

Challenges to address: Aligning schedules, pulling physicians from patient care, limited time allotted to physicians for administrative activities, competing priorities Tips: Learn more about and leverage physicians' requirements and responsibilities related to employment. Educate physicians about patient care issues in the ED.

Knowledge about physician employment arrangements (responsibilities, accountability, compensation) is helpful to gain physician participation. 


\section{Share Data \& Goals}

- Use these to gain physician buy-in for need for improvement

- Provide physician-specific feedback and support for low performers

- Use meetings or huddles to share this information

- Share information outside of ED - especially with other physician specialties that impact ED patient care issues or metrics

\section{Share Success}

- Share accomplishments to inspire physicians to participate

- Support the use of Appreciative Inquiry to build on what works well

- Use meetings or huddles to share this information

- Include hospital leadership

Challenges to address: Lack of needed resources, lack of leadership support, lack of physician investment or loyalty, culture, resistance to change

Tip: Address issues through the lens of providing better patient care instead of just discussing metrics or data

Physicians should be educated about organizational and ED goals, patient care issues or problems, and how they impact them.

\section{Include Goals in Physician Contracts}

- Gain hospital leadership engagement and support as they will be required

- Relate physician requirements, accountability, and or compensation to ED metrics as well as larger hospital goals

Challenges to address: Lack of needed resources, lack of leadership support 
Tips: Explain the importance of physician engagement in efforts to improve patient care in the ED to gain leadership buy-in. Give specific example of how physicians impact ED problems or issues. Learn more about physician employment arrangements for other physician specialties whose work impacts the ED.

Aligning ED, hospital, and physician goals is invaluable
for gaining physician engagement.




\section{REFERENCES}

Baathe, F., \& Norback, L.E. (2013). Engaging physicians in organisational improvement work. Journal of Health Organization and Management, 27(4), 479-497. doi:10.1108/JHOM02-2012-0043

Bagnasco, A., Tubino, B., Piccotti, E., Rosa, F., Aleo, G., Di Pietro, P.,...Emergency and Urgency Department of the IRCCS Giannina Gaslini. (2013). Identifying and correcting communication failures among health professionals working in the emergency department. International Emergency Nursing, 21(3), 168-172. doi:10.1016/j.ienj.2012.07.005

Berwick, D. M., Nolan, T.W., \& Whittington, J. (2008). The triple aim: Care, health, and cost. Health Affairs (Project Hope), 27(3), 759-769. doi:10.1377/hlthaff.27.3.759

Barker, K., Bosco, C., \& Oandasan, I. (2005). Factors in implementing interprofessional education and collaborative practice initiatives: Findings from key informant interviews. Journal of Interprofessional Care, 19, 166-176. doi: 10.1080/13561820500082974

Byrnes, J. (2015). Great physician engagement is key to great quality. Physician Leadership Journal, 2(2), 40-42. Retrieved from http://libproxy.lib.unc.edu/login?url=http://search.proquest.com.libproxy.lib.unc.edu/doc view/1699524539?accountid=14244

Cameron, K.A., Engel, K.G., McCarthy, D.M., Buckley, B.A., Mercer Kollar, L.M., Donlan, S.M., . . . Adams, J.G. (2010). Examining emergency department communication through a staff-based participatory research method: Identifying barriers and solutions to meaningful change. Annals of Emergency Medicine, 56(6), 614-622. doi:10.1016/j.annemergmed.2010.03.017

Centers for Disease Control and Prevention (CDC). (2017). FastStats: Emergency department visits. Retrieved February 12, 2017 from https://www.cdc.gov/nchs/fastats/emergencydepartment.htm

Chisholm, C.D., Collison, E.K., Nelson, D.R., \& Cordell, W.H. (2000). Emergency department workplace interruptions: Are emergency physicians "interrupt-driven" and "multitasking"? Academic Emergency Medicine: Official Journal of the Society for Academic Emergency Medicine, 7(11), 1239-1243. doi: 10.1111/j.15532712.2000.tb00469.x

Coiera, E.W., Jayasuriya, R.A., Hardy, J., Bannan, A., \& Thorpe, M.E. (2002). Communication loads on clinical staff in the emergency department. The Medical Journal of Australia, 176(9), 415-418. doi: coi10481_fm

Cosby, K.S. (2003). A framework for classifying factors that contribute to error in the emergency department. Annals of Emergency Medicine, 42(6), 815-823. doi:10.1016/S0196064403006358 
Cosby, K.S., Roberts, R., Palivos, L., Ross, C., Schaider, J., Sherman, S., . . S Scott, R.D., 2nd. (2008). Characteristics of patient care management problems identified in emergency department morbidity and mortality investigations during 15 years. Annals of Emergency Medicine, 51(3), 251-61, 261.e1. doi: S0196-0644(07)01252-8

Cote, G., Lauzon, C., \& Kyd-Strickland, B. (2008). Environmental scan of interprofessional collaborative practice initiatives. Journal of Interprofessional Care, 22(5), 449-460. doi: 10.1080/13561820802210950

Creswick, N., Westbrook, J.I., \& Braithwaite, J. (2009). Understanding communication networks in the emergency department. BMC Health Services Research, 9, 247-6963-9-247. doi:10.1186/1472-6963-9-247

Cross, R., \& Sproull, L. (2004). More than an answer: Information relationships for actionable knowledge. Organization Science, 15(4), 446; 446. doi: 10.1287/orsc.1040.0075

Duberman, T., Hemker, B., \& Solomon, L. (2015). Innovative strategies for physician partnerships. one health system shares the steps it took to strengthen physician engagement. Healthcare Executive, 30(3), 84-86. Retrieved from http://www.tldgroupinc.com/uploads/Healthcare_Executive_-_May-June_2015__OPR_Article.pdf

Eisenberg, E.M., Murphy, A.G., Sutcliffe, K., Wears, R., Schenkel, S., Perry, S., \& Vanderhoef, M. (2005). Communication in emergency medicine: implications for patient safety. Communication Monographs, 72(4), 390-413. doi: 10.1080/03637750500322602

Eisenberg, E.M., Baglia, J., \& Pynes, J.E. (2006). Transforming emergency medicine through narrative: Qualitative action research at a community hospital. Health Communication, 19(3), 197-208. doi:10.1207/s15327027hc1903_2

Fairbanks, R.J., Bisantz, A.M., \& Sunm, M. (2007). Emergency department communication links and patterns. Annals of Emergency Medicine, 50(4), 396-406. doi: S01960644(07)00357-5

Fitzgerald, A., \& Teal, G. (2003). Health reform, professional identity and occupational subcultures: The changing interprofessional relations between doctors and nurses. Contemporary Nurse: A Journal for the Australian Nursing Profession, 16(1-2), 9-19. doi:10.5172/conu.16.1-2.9

Flowerdew, L., Brown, R., Russ, S., Vincent, C., \& Woloshynowych, M. (2012). Teams under pressure in the emergency department: An interview study. Emergency Medicine Journal: EMJ, 29(12), e2-2011-200084. Epub 2011 Dec 20. doi:10.1136/emermed-2011200084

Flowerdew, L., Brown, R., Vincent, C., \& Woloshynowych, M. (2012). Identifying nontechnical skills associated with safety in the emergency department: A scoping review of the literature. Annals of Emergency Medicine, 59(5), 386-394. doi:10.1016/j.annemergmed.2011.11.021 
Fordyce, J., Blank, F.S., Pekow, P., Smithline, H.A., Ritter, G., Gehlbach, S., . . Henneman, P. L. (2003). Errors in a busy emergency department. Annals of Emergency Medicine, 42(3), 324-333. doi:10.1067/mem.2003.271

Gittell, J. H. (2001). Supervisory span, relational coordination, and flight departure performance: A reassessment of postbureaucracy theory. Organization Science, 12(4), 468-483.

Retrieved from http://www.jstor.org/stable/3085983

Gittell, J.H. (2003). The Southwest airlines way. New York, NY: McGraw-Hill.

Gittell, J.H. (2006). Relational coordination: coordinating work through relationships of shared goals, shared knowledge and mutual respect. In Kyriakidou, O. \& Ozbilgin, M.F. (Eds.), Relational perspectives in organizational studies (pp. 74-94). Cheltenham, UK: Edward Elgar.

Gittell, J.H. (2008). Relationships and resilience. Journal of Applied Behavioral Science, 44(1), 25-47. doi:10.1177/0021886307311469

Gittell, J.H. (2009). High performance healthcare. New York, NY: McGraw-Hill.

Gittell, J.H. (2011). Relational coordination: guidelines for theory, measurement and analysis. Relational Coordination Research Collaborative. Retrieved on September 25, 2015 from http://rcrc.brandeis.edu/downloads/Relational_Coordination_Guidelines_8-25-11.pdf

Gittell, J.H. (2012). New directions for relational coordination theory. In Spreitzer, G.M. \& Cameron, K.S. (Eds.), The Oxford Handbook of Positive Organizational Scholarship (pp. 400-411). doi:10.1093/oxfordhb/9780199734610.013.0030

Gittell, J.H., Fairfield, K. M., Bierbaum, B., Head, W., Jackson, R., Kelly, M., . . Zuckerman, J. (2000). Impact of relational coordination on quality of care, postoperative pain and functioning, and length of stay: A nine-hospital study of surgical patients. Medical Care, 38(8), 807-819. Retrieved from

https://auth.lib.unc.edu/ezproxy_auth.php?url=http://search.ebscohost.com/login.aspx?dir ect $=$ true $\& \mathrm{db}=$ rzh\&AN=2000071225\&site=ehost-live\&scope $=$ site

Gittell, J.H., Godfrey, M., \& Thistlethwaite, J. (2013). Interprofessional collaborative practice and relational coordination: improving healthcare through relationships. Journal of Interprofessional Care, 27(3), 210-213. doi:10.3109/13561820.2012.730564

Gittell, J.H., Weinberg, D.B., Bennett, A.L., \& Miller, J.A. (2008). Is the doctor in? A relational approach to job design and the coordination of work. Human Resource Management, 47(4), 729-755. doi:10.1002/hrm.20242

Greenwald, P.W., Estevez, R.M. Clark, S., Stern, M.E., Rosen, T., Flomenbaum, N. (2015). The emergency department as the primary source of hospital admission for older (but not younger) adults, American Journal of Emergency Medicine (online), doi: 10.1016/j.ajem.2015.05.041 
Guthrie, M. (2005). Engaging physicians in performance improvement. American Journal of Medical Quality: The Official Journal of the American College of Medical Quality, 20(5), 235-238. doi:20/5/235

Hartgerink, J.M., Cramm, J.M., Bakker, T., J.E.M., Eijsden, R., A.M., Mackenbach, J.P., \& Nieboer, A.P. (2014). The importance of relational coordination for integrated care delivery to older patients in the hospital. Journal of Nursing Management, 22(2), 248256. doi:10.1111/j.1365-2834.2012.01481.x

Herbert, C.P., Bainbridge, L., Bickford, J., Baptiste, S., Brajtman, S., Dryden, T., . . S Solomon, P. (2007). Factors that influence engagement in collaborative practice: How 8 health professionals became advocates. Canadian Family Physician Medecin De Famille Canadien, 53(8), 1318-1325. doi:53/8/1318

Herring, A.A., Ginde, A.A., Fahimi, J., Alter, H.J., Maselli, J.H., Espinola, J.A., . . Camargo, C. A., Jr. (2013). Increasing critical care admissions from U.S. emergency departments, 2001-2009. Critical Care Medicine, 41(5), 1197-1204.

doi:10.1097/CCM.0b013e31827c086f

Hitchcock, M., Gillespie, B., Crilly, J., \& Chaboyer, W. (2014). Triage: An investigation of the process and potential vulnerabilities. Journal of Advanced Nursing, 70(7), 1532-1541. doi:10.1111/jan.12304

Hojat, M., Nasca, T.J., Cohen, M.J., Fields, S.K., Rattner, S.L., Griffiths, M., . . Garcia, A. (2001). Attitudes toward physician-nurse collaboration: A cross-cultural study of male and female physicians and nurses in the United States and Mexico. Nursing Research, 50(2), 123-128. doi: 10.1097/00006199-200103000-00008

Institute of Medicine (IOM). (2007). Hospital-based emergency care at the breaking point. Washington, DC: The National Academies Press.

Jacobsson, M., Hargestam, M., Hultin, M., \& Brulin, C. (2012). Flexible knowledge repertoires: Communication by leaders in trauma teams. Scandinavian Journal of Trauma, Resuscitation and Emergency Medicine, 20, 44-7241-20-44. doi:10.1186/1757-7241-2044

Juarez, A., Gacki-Smith, J., Bauer, M.R., Jepsen, D., Paparella, S., VonGoerres, B., \& MacLean, S. (2009). Barriers to emergency departments' adherence to four medication safetyrelated joint commission national patient safety goals. Joint Commission Journal on Quality and Patient Safety / Joint Commission Resources, 35(1), 49-59. doi: http://dx.doi.org/10.1016/S1553-7250(09)35008-4

Kaissi, A. (2014). Enhancing physician engagement: An international perspective. International Journal of Health Services: Planning, Administration, Evaluation, 44(3), 567-592. doi: http://dx.doi.org/10.2190/HS.44.3.h 
Kilner, E., \& Sheppard, L.A. (2010). The role of teamwork and communication in the emergency department: A systematic review. International Emergency Nursing, 18(3), 127-137. doi:10.1016/j.ienj.2009.05.006

Klein, K.J., Ziegert, J.C., Knight, A.P., \& Xiao, Y. (2006). Dynamic delegation: Shared, hierarchical, and deindividualized leadership in extreme action teams. Administrative Science Quarterly, 51(4), 590-621. doi: 10.2189/asqu.51.4.590

Kreindler, S.A., Dowd, D.A., Dana Star, N., \& Gottschalk, T. (2012). Silos and social identity: The social identity approach as a framework for understanding and overcoming divisions in health care. The Milbank Quarterly, 90(2), 347-374. doi:10.1111/j.14680009.2012.00666.x

Kreindler, S.A., Larson, B.K., Wu, F.M., Gbemudu, J.N., Carluzzo, K.L., Struthers, A., . . . Fisher, E.S. (2014). The rules of engagement: Physician engagement strategies in intergroup contexts. Journal of Health Organization and Management, 28(1), 41-61. doi:10.1108/JHOM-02-2013-0024

Liebhaber, A., Draper, D.A., \& Cohen, G.R. (2009). Hospital strategies to engage physicians in quality improvement. Issue Brief (Center for Studying Health System Change),127, 1-4.

Lindgren, A., Baathe, F., \& Dellve, L. (2013). Why risk professional fulfilment: A grounded theory of physician engagement in healthcare development. The International Journal of Health Planning and Management, 28(2), e138-57. doi:10.1002/hpm.2142

Lutfiyya, M.N., Brandt, B., Delaney, C., Pechacek, J., \& Cerra, F. (2015). Setting a research agenda for interprofessional education and collaborative practice in the context of United States health system reform. Journal of Interprofessional Care, 1-8. doi:10.3109/13561820.2015.1040875

Manev, I.M., \& Stevenson, W.B. (2001). Balancing ties: Boundary spanning and influence in the organization's extended network of communication. The Journal of Business Communication, 38(2), 183-205. doi: 10.1177/002194360103800203

Manojlovich, M. (2010). Nurse/physician communication through a sensemaking lens: shifting the paradigm to improve patient safety. Medical Care, 48(11), 941-946.

doi:10.1097/MLR.0b013e3181eb31bd

Manski-Nankervis, J., Blackberry, I., Young, D., O'Neal, D., Patterson, E., \& Furler, J. (2014). Relational coordination amongst health professionals involved in insulin initiation for people with type 2 diabetes in general practice: An exploratory survey. BMC Health Services Research, 14(1), 515-515. doi:10.1186/s12913-014-0515-3

Meleis, A.I. (2016). Interprofessional education: A summary of reports and barriers to recommendations. Journal of Nursing Scholarship: An Official Publication of Sigma Theta Tau International Honor Society of Nursing / Sigma Theta Tau, 48(1), 106-112. doi:10.1111/jnu.12184 
Miles, M.B., Huberman, A.M., \& Saldana, J. (2014). Qualitative Data Analysis: A Methods Sourcebook. Los Angeles, CA: Sage.

Minamizono, S., Hasegawa, H., Hasunuma, N., Kaneko, Y., Motohashi, Y., \& Inoue, Y. (2013). Physician's perceptions of interprofessional collaboration in clinical training hospitals in northeastern Japan. Journal of Clinical Medicine Research, 5(5), 350-355.

doi:10.4021/jocmr1474w

Molesworth, B.R.C. \& Estivalb, D. (2015). Miscommunication in general aviation: The influence of external factors on communication errors. Safety Science, 73, 73-70. doi:10.1016/j.ssci.2014.11.004

Morgan, S., Pullon, S., \& McKinlay, E. (2015). Observation of interprofessional collaborative practice in primary care teams: An integrative literature review. International Journal of Nursing Studies, 52(7), 1217-1230. doi:10.1016/j.ijnurstu.2015.03.008

Mullins, P.M. \& Pines, J.M. (2014). National ED crowding and hospital quality: Results from the 2013 hospital compare data. The American Journal of Emergency Medicine, 32(6), 634-639. http://dx.doi.org/10.1016/j.ajem.2014.02.008

Murphy, A.G. (2001). The flight attendant dilemma: An analysis of communication and sensemaking during in-flight emergencies. Journal of Applied Communication Research: JACR, 29(1), 30-53. doi:10.1080/00909880128100

Parsons, M.L., Cornett, P.A., \& Burns, A.L. (2005). A healthy emergency department workplace: The staff describe it. Topics in Emergency Medicine, 27(3), 198-205. Retrieved from https://auth-lib-uncedu.libproxy.lib.unc.edu/ezproxy_auth.php?url=http://search.ebscohost.com.libproxy.lib. unc.edu/login.aspx?direct=true\&db=rzh\&AN=2009029564\&site=ehost-live\&scope=site

Patterson, P.D., Pfeiffer, A.J., Weaver, M.D., Krackhardt, D., Arnold, R.M., Yealy, D.M., \& Lave, J. R. (2013). Network analysis of team communication in a busy emergency department. BMC Health Services Research, 13(1), 109-109. doi:10.1186/1472-6963-13109

Pham, J.C., Story, J.L., Hicks, R.W., Shore, A.D., Morlock, L.L., Cheung, D.S., . . Pronovost, P.J. (2011). National study on the frequency, types, causes, and consequences of voluntarily reported emergency department medication errors. The Journal of Emergency Medicine, 40(5), 485-492. doi:10.1016/j.jemermed.2008.02.059

Pilon, B.A., Ketel, C., Davidson, H.A., Gentry, C.K., Crutcher, T.D., Scott, A.W., . . Rosenbloom, S.T. (2015). Evidence-guided integration of interprofessional collaborative practice into nurse managed health centers. Journal of Professional Nursing: Official Journal of the American Association of Colleges of Nursing, 31(4), 340-350. doi:10.1016/j.profnurs.2015.02.007 
Pines, J.M., Mullens, P.M., Cooper, J.K., Feng, L.B., \& Roth, K.E. (2012). National trends in emergency department use, care patterns, and quality of care of older adults in the United States. Journal of the American Geriatrics Society (JAGS), 61(1), 12-17. doi: 10.1111/jgs.12072

Poochikian-Sarkissian, S., Hunter, J., Tully, S., Lazar, N.M., Sabo, K., \& Cursio, C. (2008). Developing an innovative care delivery model: Interprofessional practice teams. Healthcare Management Forum, 21(1), 6-18. doi: 10.1016/S0840-4704(10)60123-8

Pruitt, C.M., \& Liebelt, E.L. (2010). Enhancing patient safety in the pediatric emergency department: Teams, communication, and lessons from crew resource management. Pediatric Emergency Care, 26(12), 942-948. doi:10.1097/PEC.0b013e3181fec9cf

Rasmussen, K., Pedersen, A.H., Pape, L., Mikkelsen, K.L., Madsen, M.D., \& Nielsen, K.J. (2014). Work environment influences adverse events in an emergency department. Danish Medical Journal, 61(5), A4812. doi: A4812

Reddy, M., \& Spence, P.R. (2006). Finding answers: Information needs of a multidisciplinary patient care team in an emergency department. AMIA ...Annual Symposium Proceedings / AMIA Symposium.AMIA Symposium, 649-653. doi:85447

Redfern, E., Brown, R., \& Vincent, C.A. (2009). Identifying vulnerabilities in communication in the emergency department. Emergency Medicine Journal: EMJ, 26(9), 653-657. doi:10.1136/emj.2008.065318

Relational Coordination Research Collaborative (2015). Brandeis University, Heller School for Social Policy and Management. Retrieved at http://rcrc.brandeis.edu/index.html

Risser, D.T., Rice, M.M., Salisbury, M.L., Simon, R., Jay, G.D., \& Berns, S.D. (1999). The potential for improved teamwork to reduce medical errors in the emergency department. The MedTeams research consortium. Annals of Emergency Medicine, 34(3), 373-383. doi: S0196064499003133

Rosenstein, A.H. (2015). Strategies to enhance physician engagement. The Journal of Medical Practice Management: MPM, 31(2), 113-116. Retrieved from http://libproxy.lib.unc.edu/login?url=http://search.proquest.com.libproxy.lib.unc.edu/doc view/1736913744? accountid=14244

Sears, N. (2011). 5 strategies for physician engagement. Healthcare Financial Management: Journal of the Healthcare Financial Management Association, 65(1), 78-82.

Shanafelt, T.D., \& Noseworthy, J.H. (2017). Executive leadership and physician well-being: Nine organizational strategies to promote engagement and reduce burnout. Mayo Clinic Proceedings, 92(1), 129-146. doi: S0025-6196(16)30625-5 
Skillman, M., Cross-Barnet, C., Singer, R.F., Ruiz, S., Rotondo, C., Ahn, R., . . Moiduddin, A. (2017). Physician engagement strategies in care coordination: Findings from the Centers for Medicare \& Medicaid services' health care innovation awards program. Health Services Research, 52(1), 291-312. doi:10.1111/1475-6773.12622

Slade, D. (2015). Communicating in hospital emergency departments. Communicating in hospital emergency departments (pp. 1; 1-158; 158). Berlin, Heidelberg: Springer Berlin Heidelberg.

Spaulding, A., Gamm, L., \& Menser, T. (2014). Physician engagement: Strategic considerations among leaders at a major health system. Hospital Topics, 92(3), 66-73. doi:10.1080/00185868.2014.937970

Spencer, R., Coiera, E., \& Logan, P. (2004). Variation in communication loads on clinical staff in the emergency department. Annals of Emergency Medicine, 44(3), 268-273. doi:10.1016/S0196064404004147

Stark, R. (2014). Increasing physician engagement: Start with what's important to physicians. The Journal of Medical Practice Management: MPM, 30(3), 171-175. Retrieved from http://libproxy.lib.unc.edu/login?url=http://search.proquest.com.libproxy.lib.unc.edu/doc view/1644486294?accountid=14244

Stevens, G.W. (2013). Engaging employed physicians: reconceptualizing the role of collective identification. Advances in Health Care Management, 15, 185-209. doi: 10.1108/S14748231(2013)0000015013

Suelflow, E. (2016). Systematic literature review: An analysis of administrative strategies to engage providers in hospital quality initiatives. Health Policy and Technology, 5(1), 2 17. doi: http://dx.doi.org/10.1016/j.hlpt.2015.10.001

Swensen, S., Kabcenell, A., \& Shanafelt, T. (2016). Physician-organization collaboration reduces physician burnout and promotes engagement: The Mayo Clinic experience. Journal of Healthcare Management / American College of Healthcare Executives, 61(2), 105-127. Retrieved from http://libproxy.lib.unc.edu/login?url=http://search.proquest.com.libproxy.lib.unc.edu/doc view/1812639924?accountid=14244

Taitz, J.M., Lee, T.H., \& Sequist, T.D. (2012). A framework for engaging physicians in quality and safety. BMJ Quality \& Safety, 21(9), 722-728. doi:10.1136/bmjqs-2011-000167

Tang, C.J., Chan, S.W., Zhou, W.T., \& Liaw, S.Y. (2013). Collaboration between hospital physicians and nurses: An integrated literature review. International Nursing Review, 60(3), 291-302. doi:10.1111/inr.12034

Tong, A., Sainsbury, P., \& Craig, J. (2007). Consolidated criteria for reporting qualitative research (COREQ): a 32-item checklist for interviews and focus groups. International Journal for Quality in Health Care, 19(6), 349-357. doi:10.1093/intqhc/mzm042 
Tremblay, D., Drouin, D., Lang, A., Roberge, D., Ritchie, J., \& Plante, A. (2010). Interprofessional collaborative practice within cancer teams: Translating evidence into action. A mixed methods study protocol. Implementation Science: IS, 5, 53-5908-5-53. doi:10.1186/1748-5908-5-53

Varjoshani, N.J., Hosseini, M.A., Khankeh, H.R., \& Ahmadi, F. (2014). Tumultuous atmosphere (physical, mental), the main barrier to emergency department inter-professional communication. Global Journal of Health Science, 7(1), 144-153. doi:10.5539/gjhs.v7n1p144

Ward, M.M., Baloh, J., Zhu, X., \& Stewart, G.L. (2016). Promoting action on research implementation in health services framework applied to TeamSTEPPS implementation in small rural hospitals. Health Care Management Review, doi:10.1097/HMR.0000000000000086

Weiss, A.J., Wier, L.M., Stocks, C., \& Blanchard, J (2014). Overview of Emergency Department Visits in the United States, 2011. HCUP Statistical Brief \#174. June 2014. Agency for Healthcare Research and Quality, Rockville, MD. Retrieved on March 5, 2016 from http://www.hcup-us.ahrq.gov/reports/statbriefs/sb174-Emergency-Department-VisitsOverview.pdf.

Whitehead, C. (2007). The doctor dilemma in interprofessional education and care: How and why will physicians collaborate? Medical Education, 41(10), 1010-1016. doi: MED2893

Whitlock, D.J., \& Stark, R. (2014). Understanding physician engagement - and how to increase it. Physician Leadership Journal, 1(1), 8-12. Retrieved from http://libproxy.lib.unc.edu/login?url=http://search.proquest.com.libproxy.lib.unc.edu/doc view/1699520227?accountid=14244

Woloshynowych, M., Davis, R., Brown, R., \& Vincent, C. (2007). Communication patterns in a UK emergency department. Annals of Emergency Medicine, 50(4), 407-413. doi: S01960644(07)01383-2

World Health Organization (2010). Framework for action on interprofessional education and collaborative practice. Geneva: WHO. Retrieved September 5, 2015 from http://www.who.int/hrh/resources/framework_action/en/

Zwarenstein, M., Goldman, J., \& Reeves, S. (2009). Interprofessional collaboration: Effects of practice-based interventions on professional practice and healthcare outcomes. Cochrane Database of Systematic Reviews, 3(CD000072), 1-30. doi: 10.1002/14651858.CD000072.pub2 